\title{
Viva Caporetto! et le mythe malapartien des origines : résonances de Marinetti, Gobetti et Emerson (1921-1923)
}

Viva Caporetto! e il mito malapartiano delle origini: echi di Marinetti, Gobetti e Emerson (1921-1923)

Viva Caporetto! and Malaparte's Myth of Origins: Echoes of Marinetti, Gobetti and Emerson (1921-1923)

\section{Emmanuel Mattiato}

\section{OpenEdition}

\section{Journals}

Édition électronique

URL : http://journals.openedition.org/cei/3329

DOI : 10.4000/cei.3329

ISSN : 2260-779X

Éditeur

UGA Éditions/Université Grenoble Alpes

Édition imprimée

ISBN : 978-2-84310-370-4

ISSN : $1770-9571$

\section{Référence électronique}

Emmanuel Mattiato, «Viva Caporetto! et le mythe malapartien des origines : résonances de Marinetti, Gobetti et Emerson (1921-1923) », Cahiers d'études italiennes [En ligne], 24 | 2017, mis en ligne le 28 février 2017, consulté le 27 mars 2021. URL : http://journals.openedition.org/cei/3329 ; DOI : https:// doi.org/10.4000/cei.3329

Ce document a été généré automatiquement le 27 mars 2021. 


\section{Viva Caporetto! et le mythe malapartien des origines : résonances de Marinetti, Gobetti et Emerson (1921-1923)}

Viva Caporetto! e il mito malapartiano delle origini: echi di Marinetti, Gobetti e Emerson (1921-1923)

Viva Caporetto! and Malaparte's Myth of Origins: Echoes of Marinetti, Gobetti and Emerson (1921-1923)

\section{Emmanuel Mattiato}

Eravamo, così, ravvicinati all'aquila e alla nube, la lontana divina essenza, attraverso l'umana nostra sostanza, si legava ai prati delle vallate. Dio si congiungeva all'humus. Si toccavano attraverso noi le stelle e le rose. Ogni diverso modo di vita in noi corrispondeva ${ }^{1}$.

1 Il n'est pas aisé de fixer définitivement les grandes étapes qui ont jalonné le parcours politique de Kurt Erich Suckert, dit Curzio Malaparte ${ }^{2}$, durant la période 1921-1943. Cet itinéraire, qui commence avec ses débuts littéraires, en 1921 précisément, et suit le cours tumultueux de ses relations avec le fascisme, s'achève avec la chute tragique du régime mussolinien. Il constitue pourtant l'une des saisons les plus fécondes de sa vie intellectuelle. La question a été traitée de manière assez approfondie par Giuseppe Pardini il y a près de vingt ans ${ }^{3}$, en s'appuyant sur des documents peu exploités à l'époque : les douze volumes des archives Malaparte publiés à compte d'auteur par Edda Ronchi Suckert, la sœur de l'écrivain, entre 1991 et 1996. L'un des principaux intérêts de la biographie politique de Pardini est de focaliser son analyse sur les années 1920 et de consacrer de nombreux chapitres fouillés à l'engagement fasciste de Malaparte, en 
particulier à son activisme syndical-révolutionnaire lorsqu'il dirigeait la revue La Conquista dello Stato, reflet du fascisme intégral des provinces. Cette aile tapageuse des chemises noires s'opposait par tout moyen aux compromis avec le parlementarisme et à la normalisation institutionnelle du régime mussolinien. Le sujet étant inépuisable a priori, on ne s'attardera pas sur la théorie du syndicalisme révolutionnaire élaborée par Malaparte et l'on se bornera à rappeler qu'elle a fait de lui l'une des plumes les plus originales (quoique tard venue ${ }^{4}$ ) de ce mouvement issu des luttes ouvrières du début du $\mathrm{xx}^{\mathrm{e}}$ siècle, en rupture avec la $\mathrm{II}^{\mathrm{e}}$ Internationale socialiste ; plus encore que par Édouard Berth ou Georges Valois, dont il connaissait la doctrine, il pourrait avoir été influencé en profondeur par Georges Sorel, dont les écrits étaient alors fort répandus et abondamment débattus en Italie, notamment dans les cercles nationalistes ${ }^{5}$. Aux origines de sa pensée, avant même son inscription au Parti national fasciste, on peut distinguer non seulement l'ombre tutélaire de Sorel, mais aussi la présence, par contraste plus que par adhésion, de Piero Gobetti ou de Filippo Tommaso Marinetti. Il est également possible de relever la trace de l'écrivain transcendantaliste ${ }^{6}$ américain Ralph Waldo Emerson, maître et ami de Henry David Thoreau?. Il ne s'agit plus seulement de constater qu'entre 1921 et la marche sur Rome, Kurt Erich Suckert délaisse les idéaux pacifistes qui avaient motivé la création de "son» avant-garde culturelle, l'océanisme, connu à travers l'éphémère revue Oceanica, et s'engage dans les rangs fascistes, mais aussi de s'interroger en profondeur sur les raisons véritables de ce qui, d'un point de vue superficiel, pourrait ressembler à une spectaculaire volte-face. Il y a peut-être, en fait, une cohérence - certes fragile - qui sous-tend cette évolution idéologique a priori contradictoire. Cela dit, le critère même de "contradiction » ne nous semble pas pertinent lorsque l'on s'intéresse à l'histoire des idées dans l'entredeux-guerres. Encore prisonniers des réflexes hérités de la guerre froide et tendant à classer les intellectuels selon un schéma politique binaire droite-gauche, nous oublions, un peu par commodité, que le débat d'idées était autrement plus complexe et " désenclavé » au lendemain de la Première Guerre mondiale.

Outre les résonances intertextuelles qui confèrent une véritable épaisseur au premier ouvrage de Curzio Malaparte, la présente étude se propose également de remonter à la source de l'io narrante malapartien, ce « moi » littéraire (Malaparte-Personaggio) que l'on pourrait définir comme massif et envahissant, reflet textuel et autonome du "vrai » Malaparte, "in carne ed ossa" ", et tout aussi intrusif, provocateur et égocentrique que l'écrivain-dandy. 1921 est l'année de naissance artistique de Kurt Erich Suckert à travers Viva Caporetto!. Ce premier livre, saisi par les autorités à chacune de ses rééditions, est avant tout un pamphlet implacable contre les autorités militaires et les embusqués d'Italie, texte dont la suprême audace consiste à transformer la honte qu'a été la défaite de Caporetto en victoire du peuple des fanti, peuple de fantassins loqueteux épuisés par des années de guerre. Mais c'est aussi bien plus que cela. En effet, si par ce texte Malaparte a, pour citer Stéphanie Laporte, la «conviction que la tranchée était l'acte de naissance social du peuple ${ }^{9}$ ", c'est aussi un véritable acte de naissance individuel, non seulement en tant qu'Italien mais aussi comme homme et comme écrivain. De plus, dès les premières lignes du récit, par l'emploi de la première personne, Suckert impose d'emblée son double, ce moi souverain qui donne à toute son œuvre son caractère propre: cet io narrante omniprésent s'insinue jusque dans ses ouvrages politiques et hante encore l'autofiction des romans de la maturité. C'est bien à l'éclosion de cette auto-conscience littéraire qu'il est donné d'assister dans cet incipit 
qu'il convient d'analyser en détails. Cette émergence d'un moi littéraire coïncide avec un dialogue contrastif et transtextuel avec Marinetti et Gobetti.

\section{Contre, tout contre Marinetti}

3 Le chapitre I de Viva Caporetto! est bien celui de la genèse concomitante d'un homme et d'un peuple. En cela, l'incipit est fondamental, au sens propre: la première phrase, remarque-t-on immédiatement, est tronquée et adopte une forme parataxique trompeuse: «-Non tutti potranno leggere questo libro ${ }^{10}$.» Il faut passer cinq paragraphes pour qu'elle soit, en quelque sorte, complétée et prenne sens : « Non tutti potranno leggere questo libro, perché non tutti avranno disperato ${ }^{11}$. » Le désespoir qui gagne les vétérans, dont la jeunesse a été brisée dans le désastre de la Grande Guerre, est donc exprimé par le narrateur suivant le mode de la scission: scission syntaxique reflétant la division intérieure, scission entre les soldats et le commandement, entre le peuple et l'élite. Cette notion de fragmentation - que Stéphanie Laporte a justement rapprochée du frammentismo et des numéros d'Oceanica, la revue d'avant-garde d'orientation pacifiste que Suckert tente de lancer à la même époque ${ }^{12}-$ impose dès l'incipit un mouvement binaire, quasi schizophrénique, qui conditionne l'ensemble du récit, déterminant sa syntaxe syncopée ${ }^{13}$.

4 L'écrivain qui s'engendre est de fait « un uomo che ha lasciato la trincea assetato d'amore e di pace, ma avvelenato fin nelle radici d'odio e di disperazione ${ }^{14}$ ", suivant une succession de phases pulsionnelles d'amour et de haine typiques des traumas ayant affecté les écrivains de la Grande Guerre (qu'il suffise de citer, ici, le cas exemplaire de Blaise Cendrars). Abordant ces questions de l'identité tourmentée sous un angle plus général, et partant des canons littéraires classiques pour en arriver au premier conflit mondial et à l'entre-deux-guerres, Massimo Lucarelli a montré précisément que la guerre - indicible par nature - ne cesse de se poétiser à travers une esthétique de la scission violente, à la fois physique, morale et identitaire ${ }^{15}$. Si l'on revient sur la phrase inaugurale de Viva Caporetto!, on soulignera la définition restrictive du destinataire : «non tutti» pourront lire ce livre, ce qui sous-entend que le narrateur adresse son discours à une minorité d'hommes, à ceux qui, comme lui, ont donné leur vie en obole pour la nation ou, du moins, à ceux qui s'identifient à cette communauté. Il est inutile de revenir ici sur cette notion cruciale de " sacrifice », qui va bien au-delà du sang versé pour la patrie, tant elle constitue l'un des grands topoi malapartiens, de Viva Caporetto! à Kaputt et La pelle, tant elle plonge ses racines idéologiques dans la militance du jeune Suckert au Parti républicain italien, pétri de messianisme politique et de christologie mazzinienne, centrée sur le sacrifice d'un Christ-Peuple censé être accablé par les puissances étrangères et par l'Église ${ }^{16}$. Suivant pareille optique, l'Unité inachevée de 1861, plus que conclure le processus historique du « Risorgimento ", l'aurait entravé, voire neutralisé ; aussi, la résurrection véritable de la « Nation » ne serait-elle intégrale qu'une fois passée l'épreuve de la guerre de 1915-1918. Alors, et alors seulement, l'Italie naîtrait en tant que Peuple uni et régénéré par le sang de ses combattants ${ }^{17}$. Déjà, point la rhétorique des martyrs de la Grande Guerre qu'alimentera le fascisme par la suite, en développant le culte des caduti.

5 On sait qu'il y eut, entre 1921 et 1923, trois éditions de Viva Caporetto!, dont le titre est remplacé dès la seconde édition de 1921 (en avril) par La rivolta dei santi maledetti. Souvent, on a exagéré les remaniements du texte opérés par Malaparte entre les 
éditions de 1921 et celle de 1923. Il est indéniable qu'en 1923, certains actes de réécriture imposent un ancrage franc dans le fascisme. Néanmoins, en nous appuyant sur le texte et rien que sur lui, nous voudrions montrer que dès 1921, nous avons affaire à un essai qu'on ne saurait définir comme strictement pacifiste.

6 Aussi, si l'on dégage certains extraits et stylèmes qui le composent, il semble bien qu'il prédispose déjà son auteur à s'agréger au Fascio de Florence. On peut certes lire Viva Caporetto! comme une diatribe antimilitariste - et cette charge corrosive est incontestable et se superpose à son contraire sans crainte du paradoxe - mais son discours rejoint l'idéologie fasciste naissante en exaltant le simple soldat et le civisme du citoyen italien idéal, en n'épargnant aucune attaque contre les embusqués et les hauts commandements qui manqueraient à leur devoir, et, enfin, en ne renonçant pas à une certaine rhétorique du romantisme guerrier : c'est ce noyau dur de son expérience guerrière qui survivra tant à sa brève saison "océanique " qu'à son engagement fasciste. Il serait trop aisé, en effet, d'opposer pacifisme et bellicisme, lutte des classes et nationalisme, l'un chassant l'autre dans la biographie de l'auteur ; au contraire, nous voudrions montrer que ces deux dimensions, loin de s'exclure, cohabitent (d'ailleurs, pour Gramsci comme pour Gobetti, qu'on ne saurait qualifier de penseurs réactionnaires, la guerre mondiale avait été, par-delà les motivations troubles des interventionnistes "bourgeois ", l'occasion de franchir un cap révolutionnaire $\left.{ }^{18}\right)$. Or voici la suite de l'incipit :

Bisogna aver disceso tutti gli scalini dell'umanità per mordere alla radice stessa della vita, aver «mangiato la terra e averla trovata deliziosamente dolce» come $\mathrm{i}$ primi uomini delle leggende indiane, aver sofferto, sperato, maledetto, bisogna essere stati uomini, semplicemente umani, per poter leggere questo libro senza pregiudizio e sentirvi il sapore della vita ${ }^{19}$.

7 Dans cette longue phrase saturée de significations multiples, au moins deux remarques s'imposent: l'une philologique, et l'autre mythologique. Tout d'abord, si l'on se souvient que Viva Caporetto! naît parallèlement à la tentative malapartienne de créer l'éphémère avant-garde "océanique », on ne serait guère surpris de relever dans cette entrée en matière une référence voilée au futurisme, première avant-garde historique dont Malaparte avait fréquenté sur le tard les représentants de l'éphémère branche florentine réunie autour de Lacerba (Soffici et Papini notamment). Nous souhaiterions, très prudemment, proposer l'hypothèse suivante à partir de la morphologie de l'extrait cité, dont nous mettons ici en relief certaines articulations syntaxiques: la phrase «Bisogna aver disceso tutti gli scalini dell'umanità per mordere alla radice stessa della vita [...], bisogna essere stati uomini, semplicemente umani, per poter leggere questo libro [...] e sentirvi il sapore della vita ${ }^{20} »$ ne serait-elle pas une allusion sans doute inconsciente à Fondazione e Manifesto del Futurismo (1909), texte embryonnaire du futurisme et archétype de toute avant-garde? En effet, la phrase en question nous semble calquer formellement cette exhortation de Marinetti à ses " amis » futuristes, celle, centrale, où il dévoile par un futur verbal, et proprement emblématique, le programme que se fixe son mouvement, le but à atteindre : «Bisognerà scuotere le porte della vita per provarne i cardini e i chiavistelli ${ }^{21}$ ! » Il s'agissait ni plus ni moins de dépasser les frontières connues de la vie humaine, d'ébranler toute connaissance et tout ordre acquis. Si l'on admet cette hypothèse, les similitudes syntaxiques et lexicales que nous soulignons en italique dans les deux sources ${ }^{22}$ pourraient certes établir un lien entre l'incipit de Viva Caporetto! et cette toute première intervention au style direct de l'io narrante du Manifeste futuriste; l'un et l'autre texte ont aussi en commun une 
position nucléaire dans l'économie de leur œuvre totale ${ }^{23}$; mais le sens diffère quant au fond. Bien plus, Suckert prend le futurisme à rebours ${ }^{24}:$ si l'on remonte de deux courtes phrases l'exhortation marinettienne citée, le plus français des écrivains italiens constatait d'abord que "Finalmente, la mitologia e l'ideale mistico sono superati ", sentant poindre «la primissima aurora ${ }^{25}$ » de l'utopie futuriste; or, l'océanisme malapartien, dont on peut saisir les infinies résonances dans Viva Caporetto!, prône au contraire une aurore bien différente, l'avènement d'un être humain nouveau, épris d'absolu, d'infini, en communion avec une humanité régénérée, pacifiée : la venue de l'« uomo umano $0^{26} »$, essentielle dans le discours malapartien, est invoquée dès l'incipit à travers la récurrence du mot "uomo ". Là où Marinetti devinait l'aube de "Centauri » et $d^{\prime}$ " Angeli ${ }^{27}$ » aux contours prométhéens et artificiels, Suckert oppose la fragilité boueuse des gueux des tranchées, pour ne rien dire des traits lancés contre la civilisation industrielle et la " morte meccanica ${ }^{28}$ » qui décime les soldats. Nous sommes ici fort éloignés des élans guerriers et hygiénistes propres au futurisme, une vision radicalement opposée de la guerre étant le principale ligne de partage entre les deux écrivains. Quand Marinetti proclamait le dépassement de l'« ideale mistico ", Suckert réaffirme au contraire les vertus d'une mystique vaguement franciscaine ${ }^{29}$, du moins ouvertement chrétienne ${ }^{30}$, même s'il s'agit plutôt d'un spiritualisme aussi évanescent qu'horizontal $^{31}$.

8 Le lexème "vita ", qui sert de pivot aux assertions malapartienne et marinettienne, a une valence quasi antithétique suivant qu'il est employé par l'un ou par l'autre: le vitalisme plein de cet «orgoglio » maintes fois affirmé dans le Manifeste du fondateur du futurisme n'a que peu à voir avec la «vita " promise aux « uomini semplicemente umani $^{32}$ ", à laquelle aspire Suckert ; notons aussi que sous sa plume, "vita » est répété deux fois dans la même phrase, comme pour insister à la fois sur ce qui l'unit et le sépare de Marinetti.

9 En somme, ces similitudes morphologiques peuvent être le fruit du hasard ou d'un mimétisme inconscient, ou encore l'hommage appuyé et peut-être ironique d'un jeune homme exalté cherchant à inscrire sa première aventure littéraire dans le sillage de ses glorieux prédécesseurs, avec qui il partage une même ferveur iconoclaste. Quoi qu'il en soit, dès le deuxième numéro de sa revue Oceanica, Suckert avait placé en pleine page l'encart suivant: "Nè passatismo nè futurismo: questa è la parola d'ordine degli 'Oceanici' ${ }^{33}$ ». D'autres attaques contre le futurisme suivront et Viva Caporetto!, en filigrane, n'en finit pas d'escarmoucher les apôtres de l'« uomo meccanico dalle parti cambiabili ${ }^{34}$ ", préférant réaffirmer le sens de l'infini et de l'humain, en réaction aux tentatives futuristes de fragmentation et de destruction violentes.

Après ces préliminaires philologiques, nous en arrivons à présent aux références mythologiques de l'incipit, qui nous amèneront à croiser encore le chemin de Marinetti. Comme pour ajouter de l'épaisseur à ces résonances transtextuelles, l'allusion entre guillemets aux légendes indiennes du second paragraphe, à ces « primi uomini » dévorant symboliquement la terre douce, est tirée de l'incipit des Hommes représentatifs d'Emerson ${ }^{35}$, mais n'est pas qu'une commode similitude poétique; elle renvoie aussi à l'univers de la mythologie cosmogonique. Le lien avec la terre fait implicitement écho, dans l'ordre vertical, à la génération d'Adam à partir de la poussière primordiale dans la Genèse, et, plus horizontalement, à celle d'une humanité nouvelle dans la terre des tranchées, ces tranchées qui sont au cœur des paragraphes 3 , 4 et 5 . S'il est ici question des "premiers hommes ", souvent créés juste après les dieux 
du panthéon, on n'omettra pas de souligner que le narrateur met aussi en avant sa propre naissance :

Non è un libro di guerra, questo. È il libro di un uomo che fin dai primi giorni è entrato, come volontario, nel cerchio della guerra, a capo chino, bestemmiando (non Dio), e che ne è uscito, all'ultimo giorno, benedicendo Dio, a capo chino, come un francescano; di un uomo che ha lasciato la trincea assetato d'amore e di pace, ma avvelenato fin nelle radici d'odio e di disperazione.

È il libro di un uomo, di un uomo qualunque, che è andato in trincea, fante tra tanti, come altri va in chiesa o all'officina o al podere per la confessione o per la bisogna cotidiana.

È il libro di un uomo normale, di un uomo «in carne ed ossa» che tutto ha accettato come un sacrificio, come un dovere istintivo, che ha sfogliato la sua complessa mentalità, fino a ridurla al più semplice boccio, per poter comprendere gli umili e i primitivi con i quali frangeva il pane e divideva la paglia ${ }^{36}$.

11 Aux " primi uomini » légendaires fait donc écho, dans la suite de l'incipit, « un uomo », dont le titre même maintient le récit dans le champ du religieux préhistorique: en effet, si l'on songe de nouveau à la Genèse, 2,7, «Adam », par jeu de mot, provient d'adâmâ qui, en hébreu, signifie la terre. Le Premier Homme biblique est à la fois l'«homme» et le «terrien ». Grâce au latin qui, comme l'hébreu, associe étymologiquement homo et humus, Suckert peut faire résonner aisément et abondamment dans son texte l'homme abaissé et la terre, le sol, l'humilitée ${ }^{37}$.

Cet « uomo » de l'incipit s'exprime avec une insistance particulière à travers l'anaphore "Questo è il libro d'un uomo ${ }^{38}$ ", qui confère au texte un rythme litanique : ici, le moi du narrateur porte encore un masque, se dissimule derrière un " uomo " générique. Le moi reste à naître, se manifestant donc pour la première fois indirectement par le biais d'un artifice périphrastique : d'une synecdoque, en l'occurrence, qui semble indiquer que le narrateur-Personnage n'est pour l'heure qu'une ébauche de sujet, pétri dans le limon de la terre, dans la boue des tranchées, suivant un processus génésiaque où se fondent mythes cosmogoniques immémoriaux et naissance simultanée à la fois d'un " homme ", futur sujet d'autofiction (de fait, le je ne s'explicite qu'à la fin du premier chapitre, nous le dirons bientôt), d'un citoyen-soldat et d'une entière communauté combattante ( «la nazione armata $\left.{ }^{39} »\right)$. Il convient ici de souligner que l'idée même d'auto-engendrement apparaît, depuis les vers révolutionnaires de Victor Hugo surtout, comme l'une des spécificités de toute littérature "révoltée " assumant pleinement l'isomorphisme reliant la rupture politique inaugurée entre 1789 et 1793, et la rupture littéraire qui se dessine à partir de l'ère romantique ${ }^{40}$; à cette nuance près que Suckert ne se résout pas à rompre complètement avec l'univers du mythe. Même au plus fort des révolutions noires ou rouges, il gardera toujours un pied dans la tradition et ne sera pas tenté par les sirènes futuristes du transhumanisme. En 1921 encore, l'« uomo umanato ${ }^{41}$ » ou « uomo umano ${ }^{42}$ » malapartien en serait même l'exact contraire, et constitue, avec son exaltation de la saine vie paysanne (avec force nuances), les garde-fous d'une pensée qui, sans cesse, nourrit l'exigence d'une incarnation ${ }^{43}$.

Dans le Manifeste futuriste de 1909, en revanche, Marinetti se fait oracle de la machine et de la vie artificielle. Pour parfaire l'analogie contrastive avec l'incipit de Viva Caporetto!, on remarquera que l'écrivain milanais, tout en repoussant le passéisme de toute mystique, recourait lui aussi à une naissance initiatique, à la mort et à la renaissance d'un homme nouveau dans la boue : celle du fossé tristement célèbre dans lequel se fracasse, en 1908, sa voiture lancée à plein régime dans les rues de Milan, ce «materno fossato, quasi pieno di un'acqua fangosa! Bel fossato d'officina! Io gustai 
avidamente la tua melma fortificante, che mi ricordò la santa mammella nera della mia nutrice sudanese... ${ }^{44}$ ». Le " procréateur» du futurisme chante ici la dévoration de la terre, de la boue, prélude à sa résurrection symbolique, et cela n'est pas sans rappeler l'allusion malapartienne à ces premiers hommes des mythes indiens se repaissant de l'humus savoureux, de la Terre-Mère; acte qui permet au jeune vétéran de goûter pleinement « il sapore della vita ».

Toutefois, là encore il y a à la fois similitude de formes et différences de fond radicales. Il n'est pas fortuit, pensons-nous, que ces échos conscients ou inconscients du Manifeste, source de la modernité littéraire du $\mathrm{xx}^{\mathrm{e}}$ siècle, résonnent dans le premier ouvrage de Suckert. Tout bien considéré, dans les deux textes, en 1909 comme en 1921, il est question d'un passage initiatique conduisant à une seconde naissance. Autoengendrement prométhéen dans le cas de Marinetti et du futurisme; naissance simultanée d'un homme et d'un peuple, dans le cas de Suckert-Malaparte.

Dans les pages de son éphémère revue d'avant-garde, Oceanica, Malaparte revient sur cette genèse individuelle et collective en des termes très proches de ceux propres au futurisme et, en particulier, à son fondateur. Nous avions déjà signalé que l'écrivain toscan, dès le second numéro de la revue, prenait pour cible la première grande avantgarde du $\mathrm{xx}^{\mathrm{e}}$ siècle. Dans le numéro suivant, il publie une "Lettera ad un oceanico svizzero » - un certain Albert Müller, peut-être auteur d'ouvrages de théologie dans l'entre-deux-guerres - par laquelle il entend préciser le sens de sa révolution océanique. L'intention consiste clairement à se démarquer du futurisme mais, ce faisant, le jeune auteur - par ironie ou par déférence voilée - reformule des topoï caractéristiques des manifestes futuristes ${ }^{45}$.

La lettre dans son ensemble calque strictement les codes rhétoriques de Marinetti : emploi (maladroit) de la langue française, considérée alors comme étalon de la littérature mondiale, oscillation entre le " je " propre au fondateur de l'avant-garde et le "nous» (collectif d'artistes engagés sur de nouvelles voies dont il s'érige en représentant), rejet du monde ancien (et en particulier, dans ce texte, de la politique entendue comme basse administration des choses terrestres) et affirmation du caractère inédit de la pensée océanique. Avant d'en arriver à la partie finale de ce qui se présente comme une sorte d'approfondissement du manifeste de l'océanisme publié dans le premier numéro d'Oceanica, Suckert revient sur la genèse de son mouvement et inscrit son histoire dans celle de ses glorieux prédécesseurs, de ses aînés vociani en particulier, écrivains et peintres italiens qui avaient fait du séjour parisien une sorte de préalable nécessaire à toute création authentique. Pas même ne manque une référence obligée à Guillaume Apollinaire, poète et " pape » dont se sont réclamées la plupart des avant-gardes des années 1910 :

Qu'est-ce que l'océanisme? Une tendence ${ }^{46}[$ sic $]$. Elle est née de la guerre, ou, à mieux dire [sic], du tourment de boue et de sang de la guerre. Ayant retrouvé, dans toutes les tranchées d'Europe, le sens de la mort, qu'ils avaient perdu du temps de la vie artificielle et provisoire d'avant la guerre, quelques hommes - sortis de ce tourment comme des entrailles d'une seconde mère - se réunirent, un soir d'été, sur les bords de la Seine, en cette île Saint-Louis qui est comme le cœur du cœur de Paris : «fluctuat nec mergitur ». L'un d'eux parlait d'un mort, d'un mort qui nous était cher. (Guillaume Apollinaire venait d'être tué par la maladie du siècle). Alors, je parlai des vivants ${ }^{47}$.

17 La seconde naissance, depuis les entrailles boueuses et sanglantes de la guerre, renvoie directement à l'incipit de Viva Caporetto! et à son espace mythique si particulier. 
S'ensuit alors une longue digression-définition de l'océanisme conçu comme quête éperdue de l'infini et, dans une certaine mesure, de Dieu. Dans la dernière partie de la lettre, l'auteur -comme pour mieux souligner l'originalité de son propre mouvement - réaffirme ce qui le distingue de ses aînés futuristes. Toutefois, les différences réelles (refus de l'artificiel, des sensations, du nietzschéisme) ne devraient pas masquer les similitudes, à commencer par le refus de l'anthropomorphisme, ce que Marinetti désignait lui-même comme cette culture du moi issue de l'humanisme et du romantisme passés :

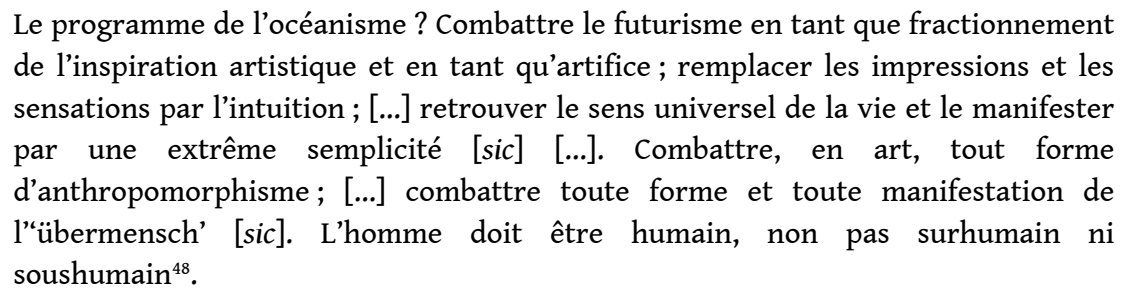

18 Les mille et une contradictions de l'océanisme semblent se concentrer dans cette lettre : de fait, tout en affirmant fièrement une volonté de dépasser les avant-gardes anciennes, Suckert ne dédaigne pas de s'approprier leurs modalités rhétoriques, certes en les détournant. Aussi, sa critique perd-elle en vigueur face à la relative inconsistance du programme océanique, qui se veut ouvert à l'«infini » et à l'« humain », tout simplement ; trop simple, en effet; trop ingénu et bien impropre à fédérer de vastes cercles artistiques; à plus forte raison lorsque se font jour les sirènes de forces politiques radicales, à vocation totalitaire et révolutionnaire. Et que dire, enfin, de l'intention malapartienne de dépasser le moi alors que Viva Caporetto!, annonce non seulement la naissance du peuple italien des vétérans, mais celle, aussi, d'un je littéraire qui, au fil des décennies, saura imposer son outrecuidance et sa démesure?

\section{Engendrement de Narcisse}

19 "Questo è il libro d'un uomo ». Comment définir cette humanité naissante et encore secrète hésitant entre je et nous, balançant entre individu et communauté combattante, elle-même en gestation? Le narrateur ne manque pas de délivrer des précisions sur cet «uomo» anonyme, défini quelques lignes plus loin comme "un uomo qualunque». L'homme quelconque, bien avant le mouvement du qualunquismo, est l'un des marqueurs des grands récits populistes, et en particulier du préfascisme (mais pas seulement): l'appel au peuple, au nom du peuple et pour le peuple est, par son ambivalence même, un mythe politique qui peut être exploité par tout groupe engagé, des dites "droites" aux diverses "gauches", et ce à l'orée du ventennio comme aujourd'hui ${ }^{49}$. Cet homme "fante tra tanti », donc, participe à l'élaboration d'un autre grand mythe politique du fascisme: l'exaltation du simple fantassin qui, par son sacrifice, donne naissance à la " Nation unie ». Dans l'imaginaire malapartien « [gli] umili e i primitivi ${ }^{50}$ ", pour citer de nouveau l'incipit, sont, en raison de leur parenté avec le monde animal et la nature édénique, les acteurs euphoriques de sa poétique personnelle et ne sauraient aucunement être réduits à un vulgaire médium de propagande. Cette figure idéalisée du simple soldat, perçu comme un "bon sauvage ", est une constante de son œuvre, que l'on songe à sa saison strapaesana $a^{51}$, à la poésie "I morti di Bligny giocano a carte ", au personnage de Calusia dans le roman Il sole è cieco, ou à la communauté populaire (féminine en particulier) et à ce même Calusia, 
ressuscité pour l'occasion, dans Il compagno di viaggio (cela est moins net à propos du peuple miséreux de Naples dans La pelle, incarnant tantôt cette pureté primitive, tantôt sa perversion extrême).

Cette croyance rousseauiste en la bonté naturelle de l'homme simple («quel carname d'ingenui e di buoni $\left.{ }^{52} »\right)$ explique aussi pourquoi, peu avant sa mort encore, Malaparte poursuit sa quête d'un Éden perdu parmi le peuple chinois sous gouvernement maoïste ${ }^{53}$. La propagande fasciste elle-même s'était nourrie de cette idéalisation du peuple, en particulier paysan et ouvrier ${ }^{54}$. Dans le cas de Malaparte, il resterait à explorer l'influence féconde que la littérature russe aurait pu avoir sur la genèse de ce mythe populaire. Les analogies avec Tolstoï (surtout), Gogol ou Dostoïevsky sont nombreuses et mériteraient une étude à part. Il ne faudrait pas non plus négliger les horizons immenses ouverts par le mouvement populiste russe ${ }^{55}$ qui, né dans la seconde moitié du xix siècle comme révolte paysanne combinant à la fois communisme des terres et traditions ancestrales du národ, présente d'étonnantes similitudes avec les conceptions politiques et agraires qu'élabore Suckert au cours des années 1920, en cherchant précisément à dépasser et la conception marxiste de la lutte des classes, et le conservatisme étriqué des traditions ${ }^{56}$.

Pour résumer, l'incipit de Viva Caporetto! proclame la naissance d'un " protosujet », d'un individu politique et fictionnel en gestation, forgé dans la tempête de 1914-1918. Au terme du premier chapitre, ce temps subjectif s'inscrit dans un temps plus vaste, suivant une lecture progressiste et émancipatrice de l'histoire héritée des Lumières ; et c'est dans cet arc temporel - en son cœur même, dirions-nous - que se manifeste pour la première fois le «je » de Malaparte-personnage, fort de sa conviction que Caporetto n'est rien moins qu'une étape fondamentale de l'histoire humaine :

Caporetto non è semplicemente un episodio militare: è una fase dell'evoluzione dell'umanità.

Il cammino che la famiglia umana percorre da secoli, attraverso nuove forme di civiltà, verso uno stato che può sembrare irraggiungibile, è segnato da pietre miliari che indicano la misura di questo sforzo di ascensione. [...] La venuta di Cristo, la Riforma, la proclamazione dei Diritti dell'Uomo, il socialismo, indicano alcune tra le principali fasi attraverso le quali la concezione umana della vita è passata.

Non a caso, né per artificio, io pongo l'episodio di Caporetto tra i fenomeni più importanti e maggiormente significativi della storia dello sviluppo intellettuale dei popoli ${ }^{57}$.

Le moi du narrateur ne se dissimule plus sous les oripeaux d'un homme quelconque et pouilleux, il s'énonce ici sans fard («io pongo »), proclame farouchement son intuition politique et philosophique. Nous croyons pouvoir dire que cette vision linéaire de l'histoire s'imposera comme une constante de la pensée politique malapartienne même après son engagement dans les rangs du fascisme, mouvement qui avait lui-même une conception historique progressiste et civilisatrice, voire utopiste, mâtinée de nationalisme et héritée à la fois de la formation socialiste de Mussolini ${ }^{58}$ et des rêveries politiques des futuristes. Selon Georges Mosse ou Emilio Gentile, cette conception du temps - ce «mito del futuro ${ }^{59}$ »- est l'un des éléments qui distinguent le plus nettement l'idéologie fasciste de l'idéologie nazie, arcboutée sur un passé fantasmé.

À la naissance de son moi fictionnel, Malaparte fait correspondre la genèse même du peuple italien : celle-ci se réalise par le sacrifice de l'infanterie, dont Viva Caporetto! décrit minutieusement la prise de " coscienza della sua funzione sociale ", conjuguant sans crainte du paradoxe nationalisme républicain et ligne marxiste-léniniste 
d'émancipation sociale. Aussi, on ne saurait scinder moi poétique et nous politique : la grande œuvre littéraire malapartienne naît de cette union entre Narcisse et la nation italienne, vivante à travers ses soldats ${ }^{60}$. Dans son œuvre, il serait vain de séparer histoire personnelle et destinée des peuples opprimés, qu'ils aient le visage du fantassin du Karst ou, quelques décennies plus tard, celui du paysan de la Chine maoïste. L'idée que l'histoire d'un peuple ou d'une classe ne peut s'écrire qu'à travers le récit concomitant d'un moi sûr de lui-même - «un personnage qui s'appelle $j e^{61}$ »deviendra en quelque sorte la marque de fabrique du roman malapartien; là encore, il se pourrait que le jeune auteur ait retenu les leçons de Ralph Waldo Emerson sur le Self, l'estime de soi ; estime de soi qui prend la forme, chez Malaparte, d'une affirmation narcissique du moi. D'Emerson, il connaissait le traité Hommes représentatifs, qui aborde indirectement ces notions; mais il se pourrait aussi qu'il ait approfondi la question à partir d'autres essais célèbres du philosophe américain ${ }^{62}$; mais nous ne voudrions pas anticiper sur notre propos.

\section{Entre libéralisme gobettien, populisme littéraire et tentation fasciste}

Comme bon nombre de théoriciens du syndicalisme révolutionnaire, Malaparte n'hésite pas à récupérer certaines analyses sociopolitiques issues de la pensée internationaliste et à les ployer à son propre nationalisme républicain. C'est sur cet aspect de Viva Caporetto! qu'il convient à présent de s'arrêter. Prenons par exemple l'interprétation très personnelle que Suckert donne de la pensée marxiste dès le premier chapitre de son pamphlet: Karl Marx, selon lui, « aveva veduto sorgere la nuova civiltà dei nostri tempi e osservati per primo, negli uomini, i segni della nuova malattia. Aveva capito che la macchina avrebbe ucciso l'anima, la religione, la tradizione. Aveva capito che la macchina avrebbe ucciso lo Stato ${ }^{63}$ ». L'économiste allemand est ici dépeint, en quelque sorte, comme un défenseur de la tradition et de l'État contre le machinisme. Notons que cette lecture hétérodoxe de la conception marxiste de l'État ${ }^{64}$ est reprise en partie par les premiers théoriciens du fascisme intégral, alors qu'elle est condamnée par la plupart des syndicalistes-révolutionnaires dont s'inspire pourtant Suckert ${ }^{65}$. Dans le second chapitre, ce dernier va plus loin, dans la mesure où, en bon militant républicain, il fustige le manque d'esprit civique de la nation italienne. Cette lutte contre l'individualisme et l'égoïsme supposés des Italiens sera aussi au cœur de la propagande fasciste, qui partage avec Suckert le souci révolutionnaire d'achever un Risorgimento interrompu par la normalisation qu'aurait imposée la maison de Savoie après 1861. Que penser par exemple, sous la plume d'un soi-disant disciple de Barbusse, de la phrase suivante, qui impose une relecture nietzschéenne des thèses d'Emerson?

La mancanza di uomini superiori, di quelli che Emerson chiama «uomini rappresentativi», capaci di tenere il popolo in un pugno di ferro o di guidarlo verso la compiutezza civile e le conquiste sociali, ha in molto aiutato l'opera disgregatrice delle varie forze operanti in seno alla giovine nazione italiana ${ }^{66}$.

Cette attente quasi messianique d'un homme politique providentiel ${ }^{67}$ peut aussi expliquer le basculement imminent de Suckert dans le fascisme, sachant que ce mouvement s'approprie des théories nées au $\mathrm{XVIII}^{\mathrm{e}}$ siècle autour des héros providentiels. Representative Men, de Ralph Waldo Emerson, date de 1850, et son inspirateur n'est autre que le Carlyle de On Heroes, Hero-Worship, and The Heroic in 
History, publié neuf ans plus tôt. Ces héros ont-ils vocation à «tenere il popolo in un pugno di ferro ", comme le suggère la lecture qu'en fait ici Suckert? Nous verrons que ce dernier finira par renier ces héros sans doute trop romantiques, leur préférant un certain césarisme, récent symptôme de la vie politique mondiale diagnostiqué par Oswald Spengler à la même époque. Cela posé, comme l'a montré Michel Onfray, il existe un fil logique entre Carlyle, Emerson, Nietzsche et Spengler; et surtout entre les trois derniers ${ }^{68}$.

Pour cette raison même, il est légitime de s'interroger sur ce messianisme politicophilosophique né en France ${ }^{69}$, qui imprègne la mythologie du "Grand Homme ", suractivée par la fascination que Napoléon, le « héros italique », exerça sur l'Europe ${ }^{70}$; un messianisme horizontal qui conduit à Carlyle, Emerson et aux déformations tardives du «surhomme» nietzschéen. Autant de syndromes de la mort de Dieu et d'une manifestation de la force typique de la modernité, autant de réservoirs mythiques donnant naissance, dans les cénacles de la jeune Italie unifiée, à un cortège de héros et de martyrs de la nation "ressuscitée " (le panthéon des Padri della Patria), censés figurer les vertus civiques du Nouvel Homme italien. Cela n'est guère surprenant si l'on considère, avec Neal Dolan ${ }^{71}$, que Carlyle et Emerson ont cherché plus que quiconque à créer de nouveaux «hommes illustres ", laïcs et paradigmatiques, au lendemain de la table rase révolutionnaire qui fit trembler l'Europe et le monde après 1789.

Dans un texte au style baroque qui singe à la fois Machiavel et D'Annunzio, le « Ritratto delle cose d'Italia " placé en prologue ${ }^{72}$ de l'édition de La rivolta dei santi maledetti publiée en 1923, Malaparte va au bout de cette logique du héros providentiel esquissée dans la première édition : il y énonce une sorte de théorie de la défaite sociale (dont la débâcle de Caporetto constituerait le modèle euphorique, dans le sillage de Custoza, Lissa et Adoua), qui provoquerait, imposerait, par réaction, l'arrivée au pouvoir du héros salvateur. L'auteur, disions-nous, pousse à ses extrêmes conséquences ce bonapartisme ${ }^{73}$, mais il finit par le renverser puisque l'homme "représentatif » n'est plus le reflet des vertus de l'homme universel, comme cela était le cas au xix siècle, mais - ironie du sort - il incarne le juste fléau, le tyran imposé au peuple en raison de l'incapacité de ce dernier à mener à terme la révolte sociale; à tel point que la condamnation de Carlyle et d'Emerson est définitive ${ }^{74}$. Ce discours de 1923 jette une lumière nouvelle sur la révolte des fantassins décrite dans l'édition originale de Viva Caporetto! et en réduit la portée révolutionnaire, dans la mesure où l'agitation de la masse - pour juste et nécessaire que soit sa colère - ne serait pas une fin en soi mais aurait besoin, pour aboutir à une transformation politique, d'être canalisée par un tyran. En somme, la révolte du fantassin et de tous les gueux des tranchées est, de toute façon, destinée à échouer parce qu'il lui manque un chef; c'est tout le sens du titre programmatique de la première partie du « Ritratto »:

Che la fatalità, quando un popolo si ribelli alla tirannia dei propri eroi legittimi e voglia far da sé, si trasporta dagli eroi negli avvenimenti, sempre contrari, e punisce in tal modo il popolo dell'orgoglio di una libertà usata in proprio danno, aiutandolo tuttavia a ritrovar così la ragione dei fatti e a sottomettersi nuovamente alla tirannia legittima degli eroi ${ }^{75}$.

Il n'est pas nécessaire, toutefois, de recourir à l'édition de 1923 pour déceler une eschatologie politique chez Malaparte et, dès 1921, il serait aisé de dresser une longue liste de citations d'inspiration parfois dannunzienne allant dans le sens la phraséologie fasciste et du messianisme nationaliste : que dire, entre autres, de celle-ci, qui déplore l'absence de sens civique des Italiens, prétexte, après 1925, à la mise en place d'un 
système éducatif totalitaire visant à la création du nouvel homme fasciste, parfait citoyen soumis à l'État ?: "La razza, la magnifica razza [italiana] che aveva dato tanti guidatori e tanti genî dal respiro immenso, non riusciva a spremere se non mediocrità e avvocati ${ }^{76}$.» Et que dire de cette Italie nouvelle qui naît dans les usines et la sueur, guidée par une élite libérale, une " esigua classe d'italiani ${ }^{77}$ » à laquelle s'identifie sans doute encore le jeune écrivain de formation républicaine qui, même après son adhésion au Fascio le 20 septembre 1922, gravitera - comme Antonio Aniante, autre fasciste hérétique $^{78}$ - dans l'entourage de Piero Gobetti, infatigable antifasciste et apôtre d'une singulière révolution libérale?

Questa Italia di oggi, turgida di forza e di attività, ansante di motori e piena degli urli delle maestranze, questa Italia di ferro e di caldaie, è l'opera di un pugno di valorosi, duri nella lotta, ostinati nello sforzo, continui nella volontà: la maggioranza della borghesia italiana è rimasta spettatrice delle doglie e del parto faticoso ${ }^{79}$.

Comprenons bien : cette Italie " piena degli urli delle maestranze » est majoritairement et implicitement prolétarienne dans le tableau qu'en donne ici l'auteur; mais elle n'évacue pas totalement la bourgeoisie; en effet, s'il reconnaît que la majorité de la bourgeoisie italienne est restée spectatrice du processus d'industrialisation, il sousentend qu'une minorité s'est associée - comme dans les tranchées - aux ouvriers afin de faire entrer la péninsule dans une nouvelle phase héroïque de son histoire. Cette élite, ce "pugno di valorosi » n'est pas sans rappeler d'abord la conception sociale d'Enrico Corradini (une majorité de bourgeois paresseux opposée à une minorité nationaliste et active) et celle aussi de Georges Sorel sur la nécessité révolutionnaire d'une union entre prolétaires et minorité capitaliste productive ${ }^{80}$; non pas que les premiers seraient au service des seconds ; bien au contraire, c'est l'inverse qui devait se produire, dans la mesure où l'industrialisation portée par une minorité de conquérants industriels aurait profité au développement de la masse ouvrière, seule capable de produire la révolution (en cela, Sorel, plus que Suckert, restait proche de Marx). On sait que dans les faits, c'est dans la petite bourgeoisie austère que va recruter le fascisme, tout en évacuant progressivement non seulement tout résidu de sorélisme ${ }^{81}$ (ce qui expliquera l'intransigeance déçue du Malaparte directeur de La Conquista dello Stato), mais aussi toute trace de ce libéralisme teinté de praxis marxiste cher à Gobetti ${ }^{82}$.

En 1922, soit un an après l'édition originale de Viva Caporetto!, lorsque Suckert assume son adhésion au Parti national fasciste, il condamne sans fard le socialisme en l'accusant précisément d'assimiler indistinctement moyenne bourgeoisie et représentants du capitalisme sauvage ${ }^{83}$. Cette défense de la bourgeoisie industrieuse est donc une ligne de continuité entre le jeune tenant de l'oceanismo et le militant inscrit au PNF. En 1922, ce dernier énonce de façon limpide la conception fasciste du rapport entre capital et travail, suivant strictement ici l'intention mussolinienne de s'approprier des catégories marxistes et de les intégrer dans la doctrine sociale du corporatisme :

Non è [...] la distruzione del capitale né la distruzione del capitalismo, che il fascismo ha per mira; ma l'integrazione di quello col lavoro, secondo un concetto di utilità bilaterale. In questo, come si vede, il fascismo si distacca dal marxismo, e in questo appunto sta la sua originalità economica. Ciò che il fascismo, attraverso il sindacalismo, mostra di poter fare, è la conciliazione tra capitale e lavoro, portando la questione sul terreno economico puro e non su quello delle rivalità e delle vendette di casta ${ }^{84}$. 

future entre prolétariat et petite bourgeoisie laborieuse, prête à lutter contre l'« imbastardirsi del tradizionale senso nostro - latino - di civiltà e di diritto ${ }^{85}$ ». En 1924, dans un texte qui, sans doute plus qu'aucun autre, révèle la proximité de vues entre Malaparte et lui, Piero Gobetti ne sauve du Risorgimento que de rares figures du processus unitaire, la plupart de ses acteurs n'étant que des héros abusant d'une creuse rhétorique; au contraire, les pragmatiques Carlo Cattaneo et Cavour apparaissent comme les seuls, aux yeux de l'intellectuel turinois, à ne point trahir l'esprit de la révolution libérale et à avoir encouragé le renouvellement des élites en instillant dans le peuple un sain esprit de compétition ${ }^{86}$.

En multipliant les références à Gobetti, nous constatons les multiples passerelles idéologiques existant entre lui et le vétéran toscan: si les extraits tirés de Viva Caporetto! cités jusqu'ici entretiennent une certaine compatibilité avec la Weltanschauung préfasciste, il est indéniable aussi qu'ils auraient pu conduire leur auteur à s'engager plus franchement dans les réseaux libéraux qu'animait son ami Piero Gobetti. Certes, Malaparte collabora épisodiquement à La Rivoluzione liberale dirigée par Gobetti (ce dernier fut même l'éditeur critique d'Italia barbara et il était aussi prévu qu'il publie le second roman de Malaparte, Viaggio agli inferni, finalement jamais édité ${ }^{87}$ ), mais l'on sait que son choix définitif se porte sur la militance au sein du Parti national fasciste. Une commune vénération critique du Risorgimento, l'idée selon laquelle la guerre de 1915-1918 conférait au peuple une conscience de classe et, enfin, le rôle particulier de guides et d'éducateurs que les intellectuels étaient destinés à jouer auprès du prolétariat auraient pu, en 1921, préparer le terrain à une franche adhésion de Malaparte à la révolution libérale promue par Gobetti. Il n'en fut rien, d'autant plus que Malaparte, dans le sillage de Sorel, nourrissait de la suspicion et du mépris pour l'intelligentsia ${ }^{88}$.

Loin d'être un élément isolé, la perspective d'une jonction entre le peuple italien, encore trop incivil, et l'élite saine de la bourgeoisie (qu'il se garde bien de confondre avec les possédants et les exploiteurs, comme le feront les marxistes) est un thème récurrent de Viva Caporetto!. Cela s'explique aisément si l'on songe que c'est de cette union que doit naitre, dans une perspective eschatologique et révolutionnaire, la nouvelle civilisation italienne. Malaparte passe du général au particulier en se mettant en scène comme intermédiaire entre ce peuple et les cercles de la culture; et de la sorte, il accomplit, au quatrième chapitre, un retour à l'incipit en évoquant la renaissance conjointe d'un peuple et d'un citoyen pétri d'idéalisme républicain :

Quando parlavo delle cose nostre, del cielo, dell'acqua, della montagna, della bufera che passava urlando e abbattendo, che spezzava le braccia e le gambe ai più disgraziati di noi, essi mi ascoltavano in silenzio [...]. Quello che io dicevo, essi pure sentivano, ma quasi bestialmente, con la semplice e chiara intuizione dei primitivi: e scoprire che un uomo, fatto come loro, non diverso da loro nella forma fisica, negli istinti e nei bisogni, nelle necessità che sono proprie dell'uomo e in certo grado proprie della bestia, trovava naturalmente le parole adatte a esprimere le loro sensazioni e i loro pensieri, a dar corpo a ciò che confusamente sentivano, a dare una ragione del mondo e delle cose umane, era per loro cagione di meraviglia e di gioia.

Ed essi m'insegnavano come l'uomo è capace di crearsi una nuova anima e una nuova vita ${ }^{89}$.

La communion entre le peuple d'en bas et une élite bourgeoise prend dans cette citation les contours d'une expérience intime et traduit en des termes spiritualistes le 
renouvellement profond de l'âme et de l'existence entières; la référence à l'intuition est une catégorie à la fois émersonienne et bergsonienne sur laquelle il faudra revenir. Le poète-soldat (et narrateur) sert ici d'intermédiaire entre le monde élémentaire et un peuple analphabète, il exprime culturellement par sa parole ce que sentent naturellement les fantassins non instruits. Cette affinité de pensée dépassant les clivages entre classes sociales est celle, peut-être fantasmée, que cultive un jeune bourgeois lettré qui se souvient que, dans sa jeunesse, il fut placé en nourrice dans une famille ouvrière, celle du légendaire Milziade Baldi, dans sa ville natale de Prato. Cette espérance d'une union fraternelle entre le prolétariat (terme sur lequel insiste Suckert quand il mentionne les fantassins) et une élite laborieuse et modeste, traduit moins ici un discours fasciste (même si de nombreux fascistes ont partagé cet espoir d'un consensus populaire dépassant les classes sociales ${ }^{90}$ ). Toutefois, on dénombre maints stylèmes (pré)fascistes plus radicaux dans les pages de Viva Caporetto!, qui contredisent l'image d'un Malaparte potentiellement gobettien ou, stricto sensu, pacifiste.

La critique de la démocratie parlementaire est un marqueur notable du discours des premiers Fasci, et Suckert se plaît à dénoncer «la democrazia invadente - sudicia democrazia latina che invece d'insegnare il rispetto per la collettività insegnava a odiare le maggioranze e a ridere dello stato ${ }^{91} »$. La critique de l'incivilité dont seraient coupables les masses italiennes, par manque d'éducation politique, donne lieu encore à une étrange diatribe contre les émigrés italiens: le sommet de "l'abbiezione a cui erano scese le nostre masse di emigranti » devrait sans doute être identifié dans leur « rancore verso la patria » et « la nostra bandiera ${ }^{92}$ ». Sous sa plume, en outre, la guerre italo-turque de 1911 aurait été imposée «dalla nostra insostenibile situazione mediterranea ${ }^{93}$ ", ce qui est pour le moins surprenant de la part d'un pseudo pacifiste ; et que penser du chapitre III, focalisé sur une critique acerbe des dix mois de neutralité italienne au début du conflit mondial, ou d'autres passages qui chantent « il magnifico sforzo della nostra guerra ${ }^{94}$ » ou l'italianité du peuple, son attachement aux valeurs traditionnelles ${ }^{95}$ ?

Dans les pages du premier numéro de la revue Oceanica fondée par Suckert, une semblable exaltation de l'italianité et du "génie de la race» allait de pair avec l'affirmation de l'origine italienne de l'avant-garde océanique et contenait, en contrechamp, une mise à distance des «étrangers ${ }^{96}$ ». Et ce n'est là qu'un partiel éventail. Cela doit-il nous surprendre? Ces questionnements du jeune et fougueux polémiste toscan, où se mêlent à la fois progressisme social et critique du parlementarisme, teintée de l'attente quasi messianique d'un chef, ne sont pas plus paradoxaux et incohérents que les appels d'un Mussolini encore socialiste (dès ses articles pour La Lotta di Classe, fondée en 1910) à renverser la démocratie d'un pays corrompu, à former une élite prolétarienne et guerrière ou à promouvoir les vertus spirituelles d'une religion civique.

\section{L'ombre d'Emerson : des « hommes représentatifs » ou un homme fort?}

Derrière les coups de boutoir de Viva Caporetto!, on assiste à la naissance d'une pensée dissidente et, parfois, peu soucieuse de cohérence doctrinale. Cela explique pourquoi, au milieu d'opinions trahissant un certain conservatisme, il est possible aussi de distinguer d'innombrables références marxistes plus orthodoxes, qui rappellent les 
meilleures pages de Barbusse : pour ne citer qu'un exemple, on songe à ce passage où le narrateur observe que les soldats du front, quel que soit leur camp, sont avant tout des prolétaires en manque d'unitép ${ }^{7}$.

Tout bien considéré, le passage de Malaparte d'un pacifisme « impur » au fascisme reste toujours une énigme. En effet, toutes ces impuretés, précisément, qui entachent son discours pacifiste contre le système ne permettent pas d'expliquer complètement ce qui l'amène, moins de deux ans après l'expérience d'Oceanica et de Viva Caporetto!, à assumer sans ciller son engagement au Fascio de Florence.

Il y aura toujours une part d'insondable mystère dans cette option politique qui, sans doute, ne cessera d'interroger les générations de chercheurs. Nous avons tenté de lever un pan du voile en identifiant des passages et des stylèmes compatibles avec une doctrine fasciste alors elle-même en voie d'élaboration. De plus, début 1921, les chemises noires sont encore loin de s'être emparées du pouvoir. Outre son opportunisme, qui pourrait expliquer la quasi-coïncidence entre la marche sur Rome et son inscription au $\mathrm{PNF}^{98}$, il semble qu'il faille interpréter son choix de militer au sein du fascisme suivant une double dialectique.

40 La première renvoie à la théorie des grands hommes ou "hommes représentatifs ", capables d'incarner le destin de tout un peuple; la seconde, qui lui est liée, passe par la conception que le jeune écrivain pouvait se faire du nationalisme, vu comme phase historique transitoire devant déboucher sur une réelle révolution sociale. Tout d'abord, concernant l'attente messianique d'un sauveur de la nation, nous avions remarqué que la perspective d'un héros capable de tenir le peuple "in un pugno di ferro" apparaissait dès $1921^{99}$, dans l'édition originale de Viva Caporetto!, et que cette dimension était accentuée dans la réédition de 1923. Toutefois, dès l'édition de 1921, les références à l'avènement d'un guide, d'un chef révolutionnaire sont loin d'être marginales. De fait, le narrateur déplorait que «il nostro miserabile popolo di lavoratori e di migratori, d'artigiani e di contadini » soit « non guidato, non curato, non educato $^{100} »$; cette foi dans l'éducation et la conduite du peuple ne doit pas nous surprendre sous la plume d'un ardent républicain. À la fin du récit, au terme du chapitre XI, on peut lire ce long passage, autrement plus significatif, qu'il faut rapporter intégralement :

Dopo le prime violenze, dopo l'orgia di sangue e di saccheggio, le moltitudini in rivolta si arrestano spaurite, ubbriache di vino e di canzoni, sbigottite dal vuoto e dal silenzio che pesano su di loro.Questo è il momento degli uomini forti: Marat, Danton, Trozki, Lenin, tutta la coorte dei guidatori, dei dittatori, dei «pastori di popolo» è balzata fuori dall'angoscia di questi momenti, armata come Minerva dal cranio di Zeus.

Impaurita dalla sua stessa violenza senza freno, la folla in rivolta si curva dinanzi all'uomo forte che la percorre tutta col suo sguardo d'imperio, che la percote col gesto, con la voce, con la volontà formidabile.

Tutte le volontà si appuntano in una sola. Dalla materia informe e diversa di una moltitudine, si forma un solo corpo d'uomo: fascio di muscoli, di nervi e di tèndini intorno a una volontà di macigno.

La crisi di tutte le rivoluzioni è in questo brusco passaggio dalla libidine sfrenata alla disciplina. Non tutte le rivoluzioni hanno compiuto questo passaggio come una evoluzione: molte come una involuzione. La crisi sta nel pericolo di un ritorno, di una reazione. Chi può leggere negli occhi dell'uomo sorto a dominare la folla?

Egli cammina diritto, volgendo le spalle agli uomini senza volontà, che lo seguono perdutamente. Egli va, senza voltarsi: ma dove ? - Durante le giornate di Caporetto 
nessun uomo era uscito dalla folla dei senza-fucile, a capeggiare il movimento di rivolta $^{101}$.

41 Dès 1921, donc, on constate que l'auteur considère que la révolte du peuple reste insuffisante, qu'elle exige l'action transformatrice d'un homme fort. Les exemples qu'il choisit de citer comme "uomini forti " sont tous issus des forces dites de gauche, suivant une ligne attendue qui unit les montagnards de 1791 aux bolcheviques de 1917. Nous sommes donc loin d'un appel à un chef qui pourrait, de près ou de loin, ressembler à un tyran réactionnaire, d'autant plus que le tableau décrit les lendemains de la "révolte " de Caporetto en 1917 et non une situation sociale située plus tard. Toutefois, on ne peut qu'être frappé par le fossé qui est ici placé entre la masse - décrite en des termes très dépréciatifs, ivre de vin, perçue comme «materia informe », incarnant la "libidine sfrenata » - et l'homme fort qui devient la tête, le chef, de cette foule indifférenciée. Mais cette apologie de l'homme de poigne s'achève par une question en suspens, qui confirme qu'aucun chef n'était prêt, en 1917 du moins, à prendre la tête de la révolte et à la transformer en révolution : « Chi può leggere negli occhi dell'uomo sorto a dominare la folla? » Cela suggère que persiste le risque d'un « ritorno, di una reazione »; et que, d'une certaine manière, l'auteur se défie de toute récupération politique de la rébellion par un homme providentiel. Le risque d'entraîner les foules vers un gouffre est aussi suggéré par la seconde interrogation qui conclut notre citation : « Egli va, senza voltarsi: ma dove?»

L'issue du « brusco passaggio dalla libidine sfrenata alla disciplina » de toute révolution est donc prudemment envisagée comme une prise de risque politique, pouvant se traduire soit par une évolution, soit par une involution, pour reprendre encore les catégories du jeune polémiste. Toutefois, au-delà de la menace d'une "réaction », la direction d'un chef semble bien être, dès l'édition de 1921, nécessaire à l'accomplissement d'un authentique processus révolutionnaire. Pour Maurizio Serra, l'attente d'un chef apte à guider le soulèvement général est non seulement présente dans Viva Caporetto!, mais révèle aussi une naturelle prédisposition au fascisme ${ }^{102}$.

Avant de méditer sur les liens unissant Malaparte à Emerson sur cette question du " grand homme ", il reste à identifier le chaînon manquant entre cette admiration pour la révolution jacobine et l'attente d'un messie politique; la doctrine nationaliste pourrait bien être le « liant » entre ces aspirations contradictoires. Quoique vaguement rattaché aux pacifistes de Clarté, organisation et revue animées par Henri Barbusse et Romain Rolland, le mouvement Oceanica (dont la brève activité coïncide avec la publication de Viva caporetto!) n'exclut pas, du moins pour son fondateur, le nationalisme. Quoique proche par bien des aspects de ses aînés français, Malaparte reste tributaire de sa militance au sein du Parti républicain italien et donc du mazzinianisme. Pour Mazzini comme pour Fichte, pour Malaparte comme pour Gramsci ${ }^{103}$, l'accès à une société internationale unie doit nécessairement passer par une phase nationale plus ou moins transitoire (clairement temporaire dans le cas de Gramsci, elle apparaît beaucoup plus problématique dans le cas des théoriciens nationalistes du XIX ${ }^{e}$ siècle). Dans son importante "Lettera a un oceanico svizzero ", publiée en février 1921, l'ardent fondateur de l'avant-garde océanique invite à :

Ne pas s'occuper de détails : seulement l'ensemble des choses doit avoir un intérêt pour nous; par conséquent, combattre tout forme "folkloriste », pour ne pas dire nationaliste, de l'art et de l'intelligence spéculative ; arriver à l'internationalisme par le nationalisme : ne pas s'arrêter aux questions particulières, aux races et aux patries, aux familles et aux mentalités ethniques [... $]^{104}$. 
Quoiqu'il puisse paraitre anodin, ce bref passage nous semble d'une importance inouïe dans la mesure où Suckert-Malaparte y affirme - outre son rejet du détail, point important sur lequel nous reviendrons et qui nous ramènera encore à Emerson - que le nationalisme n'est qu'une phase initiale de l'internationalisme et, donc, qu'il en dépend, n'étant en soi qu'un moment politique provisoire préparant l'avènement d'un État universel. Ces quelques lignes pourraient donc éclairer la conversion de Malaparte au fascisme, celui-ci s'illusionnant, à partir d'octobre 1922, sur le potentiel révolutionnaire des chemises noires et sur leur capacité à passer du particulier national à l'universel international. Par ces dernières citations - celle sur «l'uomo sorto a dominare la folla » et celle sur le nationalisme comme prélude à l'internationalisme nous voulions montrer que la pensée de Suckert contient dès 1921 des ferments idéologiques pouvant expliquer ses choix politiques futurs, sa radicalité ; et établit, en ce sens, une certaine cohérence dans son évolution générale.

Pour conclure notre recherche, il resterait à livrer quelques réflexions sur les liens entre Malaparte et Emerson, ce dernier se trouvant être le raccord entre l'idéologie horizontale et universaliste typique d'Oceanica ${ }^{105}$, et une exaltation du grand homme, dont on a montré que, par glissement, elle préparait en quelque sorte le terrain à l'«uomo forte " malapartien, capable de mener à son achèvement une révolution populaire. Commençons par citer in extenso l'incipit de l'essai Hommes représentatifs, dont nous avons dit qu'une phrase était citée entre guillemets, sans mention de l'auteur, dans l'incipit même de Viva Caporetto!, déjà amplement commenté. Le texte célèbre du philosophe et poète américain offre une clé de lecture particulière pour mieux saisir la profondeur du pamphlet de Malaparte :

Il est naturel de croire aux grands hommes. Si nous découvrions tout à coup des héros dans les compagnons de notre enfance, et s'ils nous paraissaient dans une condition royale, nous n'en serions point surpris. Toute mythologie s'ouvre par des demi-dieux, et il y a la grandeur de la poésie dans cette conception : elle signifie que leur génie est souverain. Dans les légendes de Gautama, les premiers hommes mangèrent la terre et la trouvèrent délicieusement douce.

La nature semble exister pour les excellents. Le monde est soutenu par la véracité des braves gens : ils font la terre saine. Ceux qui ont vécu avec eux ont trouvé la vie joyeuse et nourrissante. La vie n'est douce et tolérable que parce que nous croyons à une telle société; et, effectivement, ou idéalement, nous nous arrangeons pour vivre avec des supérieurs ${ }^{106}$.

Le clin d'œil de Malaparte à Emerson nous invite à rapprocher les deux incipit. Disentils la même chose? Pas exactement. Pour Emerson, l'allusion aux légendes indiennes renvoie à deux notions fondamentales de son essai, voire de toute son œuvre: d'une part, la grandeur quasi mythique des hommes « supérieurs », représentatifs; et, d'autre part, la possibilité pour le commun des mortels de profiter de leur rayonnement, de jouir (de manger) des fruits de la terre. Cette notion du goût des fruits, à la fois nectars spirituels et matériels, est un topos de la poétique émersonienne ${ }^{107}$.

Dans la relecture malapartienne de cet incipit, la perspective semble inversée : si l'on se souvient bien du début de Viva Caporetto!, l'allusion aux premiers hommes mangeant la terre douce n'est qu'un prétexte soulignant la nécessité d'un abaissement, d'un retour à la glèbe vu comme humiliation. Pouvait-il en être autrement après cette descente infernale que fut l'expérience de la Grande Guerre pour une génération entière? Toutefois, en dépit des différences, il reste que Viva Caporetto! débute comme un récit dont nous avons montré qu'il concentre en son principe une certaine charge mythique, 
qu'on pourrait partiellement rapprocher de l'esprit religieux qui imprègne les écrits d'un Emerson. Plus encore, le syncrétisme religieux qui parcourt les grands textes du philosophe et poète américain - l'on songe en particulier à Nature ou Over-Soul, sur lesquels nous reviendrons - inspire les écrits de Malaparte publiés à la même époque que Viva Caporetto!, et caractérise tout particulièrement le ton des écrits publiés dans la revue avant-gardiste Oceanica. Au-delà de Malaparte lui-même, l'influence d'Emerson s'est manifestée plus largement sur ces philosophes et poètes européens et américains (à commencer par Walt Whitman) qui, au tournant du XIXe et du $\mathrm{Xx}^{\mathrm{e}}$ siècle, placeront la quête d'une certaine mystique au cœur de leur démarche littéraire. En cela, Romain Rolland ou le fondateur d'Oceanica se situent, par leur aspiration à l'infini océanique, entre Emerson et ses émules ${ }^{108}$, futurs fers de lance de la contre-culture des années 1960-1970 109 .

Il est un concept dysphorique qui est crucial dans Viva Caporetto! et qui incarne un renversement complet de cette soif océanique d'absolu et d'infini, renversement qui s'impose comme une conséquence de la guerre des tranchées, subie comme une régression brutale de l'humanité: c'est l'idée du "fragmentaire", qui imprègne le premier et l'avant-dernier chapitre du premier livre publié par Malaparte et qui était au cœur du manifeste de l'océanisme ${ }^{110}$. Cette maladie, cette "lebbra del 'frammentario' 111 ", entraînerait ainsi les êtres à se détourner de la totalité du cosmos pour se concentrer sur d'inutiles détails. Étonnamment, le jeune auteur identifie l'origine de cette plaie morale dans l'œuvre d'éminents poètes français, sortes d'hommes représentatifs inversés, si l'on conserve la définition d'Emerson, selon laquelle l'homme supérieur doit révéler aux autres hommes l'impérissable Beauté. En effet, selon Malaparte, une "mostruosa avversione dell'infinito [...] spinge Mallarmé, Verlaine et Rimbaud, cercatori di assoluto e tre fra gli uomini più rappresentativi dell'epoca, a buttarsi in ginocchio dinanzi al 'particolare'. ("Je suis hanté! l'Azur! l'Azur! l'Azur ! l'Azur!») L'odio dell'azzurro, dell'infinito, è la peste dell'epoca' ${ }^{112}$.» D'ailleurs, tout le premier chapitre de Viva Caporetto! est une sorte de passage en revue assez maladroit des hommes représentatifs du XIX siècle entre Allemagne, France et Italie. L'auteur n'accorde d'ailleurs qu'à une sorte de trinité italienne - Mazzini, Garibaldi et Leopardi - la faculté d'avoir su dépasser le particulier pour accéder à l'universel, à l'océanique.

49 Totalité versus division, fragment: ce grand thème occupe une place centrale dans l'œuvre d'Emerson. Toute connaissance transcendante, délivrée par les Anciens ou dans les Écritures, est réputée parcellaire ; l'homme aspire à la totalité mais est prisonnier des limites de ses facultés ${ }^{113}$. Chez Emerson, il s'ensuit, dans l'optique de sa relecture du platonisme dérivée de Swedenborg, qu'il appartient à l'homme supérieur de se libérer de la conception fragmentaire du monde inhérente à l'homme fini et de se relier à l'Essence, à la « divine essence ${ }^{114}$ ", plus connue dans son œuvre sous le nom d'Over-Soul, dans le texte homonyme :

We live in succession, in division, in parts, in particles. Meantime within man is the soul of the whole; the wise silence; the universal beauty, to which every part and particle is equally related; the eternal ONE. And this deep power in which we exist and whose beatitude is all accessible to us, is not only self-sufficing and perfect in every hour, but the act of seeing and the thing seen, the seer and the spectacle, the subject and the object, are one. We see the world piece by piece, as the sun, the moon, the animal, the tree; but the whole, of which these are the shining parts, is the soul ${ }^{115}$. 
Cette aspiration de l'homme à la communion avec l'infini habite notamment le chapitre «Swedenborg» de Representative Men, mais plus encore le célèbre texte Nature, publié anonymement quatorze an plus tôt, où Emerson donne sa définition du « globe oculaire transparent », pour signifier - sous l'influence des traditions orientales ${ }^{116}$ - la fusion entre l'être et l'étant, entre le tout et le rien :

In the woods, we return to reason and faith. There I feel that nothing can befall me in life, - no disgrace, no calamity (leaving me my eyes), which nature cannot repair. Standing on the bare ground, - my head bathed by the blithe air and uplifted into infinite space, - all mean egotism vanishes. I become a transparent eyeball; I am nothing; I see all; the currents of the Universal Being circulate through me; I am part or parcel of God ${ }^{117}$.

On retrouve ces grands thèmes émersoniens dans l'Appello a tutti i ricercatori di Dio, publié dans le dernier numéro d'Oceanica :

Questo significato unico - che noi siamo abituati a non vedere, a non intuire, è il Dio che noi OCEANICI coscientemente cerchiamo e di cui tutti incoscientemente anelano partire alla ricerca. Bisogna sostituire l'intuizione alla sensazione fisica. Intuire ciò che gli altri sanno o sentono. Rinnegare le idee particolari per annegare nelle idee generali che formano l'essenza della vita. Ritrovare il senso oceanico della vita. Cioè il senso dell'infinito. Il quale è Dio ${ }^{118}$.

52 L'homme représentatif est cet homme supérieur capable de retisser le lien rompu qui existait entre le Tout et le particulier, entre l'Essence et les créatures. Cela posé, tout individu doit affirmer sa propre originalité et sa propre capacité à relier en lui-même l'Essence et la Création. Chez Emerson comme chez le fondateur d'Oceanica, l'intuition $^{119}$, supérieure à la connaissance logique, est le medium entre moi-même et le monde, entre Dieu, les autres et le moi ${ }^{120}$. À ce sujet, on se souvient qu'Emerson éprouvait de franches sympathies politiques pour les républicains européens et l'une de ses plus proches amies, Margaret Fuller, était elle-même une connaissance et une fervente admiratrice de Mazzini, si bien qu'elle appelle les États-Unis à venir en aide aux républiques italiennes de $1848^{121}$ (cette même année, en plein printemps des peuples, Emerson se rend à Paris et participe à une fête républicaine place de la Concorde). Cette anecdote, si l'on veut bien la rapporter à Malaparte, n'est pas secondaire car des écrits de Mazzini et d'Emerson émerge la figure d'un Christ social dont nous avons à plusieurs reprises remarqué la prégnance tant dans Viva Caporetto! que dans son œuvre de la maturité. Pour Emerson comme pour le Malaparte fondateur de l'océanisme, « the true Christianity, - a faith like Christ's in the infinitude of man, is $\operatorname{los}^{122} »$. L'homme, reflet du Christ émané, porte en lui l'infini (d'où l'image malapartienne de l'océan illimité1 ${ }^{123}$ ); mais cette foi soi-disant pure est perdue et ne saurait être retrouvée dans les religions institutionnalisées.

53 Il est probable que le chapitre des Hommes représentatifs qui a le plus inspiré Malaparte dans Viva Caporetto! est le sixième, consacré à Napoléon. Emerson le classe parmi les grands hommes «qui tiennent pour les faits", "les rugueux ${ }^{124}$ ", catégorie prémonitoire de cet homme fort invoqué à la fin de Viva Caporetto!, seul capable de modeler l'histoire en donnant un sens aux ruptures révolutionnaires. Que l'on se souvienne que, dix ans plus tard, dans Technique du coup d'État, Malaparte fait de Napoléon, en dépit des limites inhérentes à son époque, l'ancêtre des putschistes du $\mathrm{xx}^{\mathrm{e}}$ siècle. Chronologiquement, le futur dominateur de l'Europe est le point de départ de la réflexion malapartienne sur le Prince moderne, ce qui est loin d'être un détail à l'ère des césarismes, de spenglerienne mémoire. 
Sous la plume d'Emerson, Napoléon est l'héritier de 1789, incarne le peuple, apparaît comme le reflet de la masse, comme celui qui achève un siècle et en inaugure impérialement un autre. Le philosophe américain a très tôt perçu que le xIX siècle serait l'«âge d'or » du progrès technique, de la toute-puissance de l'argent et du commerce, suivant une lecture spiritualiste et morale de l'histoire. Bonaparte est le parangon du démocrate (le « démocrate type $\left.{ }^{125} »\right)$, dans ses vices et ses vertus :

La première classe [les conservateurs] est timide, égoïste, illibérale, haïssant l'innovation, et continuellement perdant nombre de ses membres par la mort. La seconde classe est égoïste aussi, envahissante, hardie, confiante en soi, toujours surpassant l'autre en nombre, et recrutant ses contingents d'heure en heure par les naissances. Elle désire garder toute avenue ouverte à la compétition de tous, et multiplier les avenues; - classe des hommes d'affaires en Amérique, en Angleterre, en France, et dans toute l'Europe ; classe d'industrie et d'habileté. Napoléon est son représentant ${ }^{126}$.

On peut identifier dans ces lignes les qualités de l'homme représentatif, admiré moins pour sa grandeur individuelle que pour sa capacité à représenter, à incarner son temps et les forces nouvelles à l'œuvre. Il est difficile de ne pas penser aux pages déjà citées de Viva Caporetto! où Suckert fait l'éloge des classes bourgeoises laborieuses et progressistes qui cherchent à hisser l'Italie au rang des grandes puissances modernes, de ce «popolo di soldati, di professori, di industriali, un popolo che era riuscito a concretare in lavoro, in produzione, in ricchezza il pensiero di tutti i secoli, a ridurre la metafisica in meccanica, il libero pensiero in funzionario dello stato ${ }^{127}$ ». C'est bien cette conception de l'homme représentatif qu'il ébauchait dans le long portrait susmentionné de l'«homme fort » qui fit défaut à Caporetto, lorsqu'il écrivait que "Tutte le volontà si appuntano in una sola. Dalla materia informe e diversa di una moltitudine, si forma un solo corpo d'uomo ${ }^{128}$ ». Alors certes Napoléon est aux yeux d'Emerson « un organe et un chef » pour les " masses industrieuses ${ }^{129}$ » en plein essor, mais, conformément au portrait que dressera Malaparte de l'homme fort, il se distingue aussi par sa poigne, voire par cette violence politique typique du XIX ${ }^{e}$ siècle, plus extrême encore à l'âge des totalitarismes; ce n'est pas un hasard que Malaparte cite Emerson quand il déplore l'absence, en Italie, d'« "uomini rappresentativi”, capaci di tenere il popolo in un pugno di ferro ${ }^{130} »$. Emerson décrit en effet l'empereur comme " un homme de pierre et de fer [...] ; un homme qui ne s'embarrassait d'aucun scrupule [...]. "Ma main de fer", disait-il, "n'était pas au bout de mon bras: elle était immédiatement reliée à ma tête." Il respectait le pouvoir de la nature et de la fortune, et attribuait à cela sa supériorité, au lieu de se faire valoir lui-même comme les hommes inférieurs $[. . .]^{131}$ ».

Quoiqu'il affiche une certaine admiration pour son génie politique, Emerson ne se prive pas de dresser un portrait critique du conquérant accusé d'avoir mis l'Europe et la Russie à feu et à sang. De fait, après un passage en revue des faiblesses morales du Petit Caporal, jugé égoïste, sans scrupule et violent, le chapitre s'achève sur l'affirmation de l'idéal socialiste émersonien :

Tant que notre civilisation sera essentiellement une civilisation de propriété, de barrières, d'exclusivisme, elle sera le jouet d'illusions. Nos richesses nous laisseront malades; il y aura de l'amertume dans notre rire; et notre vin nous brûlera la bouche. C'est que le seul bien qui profite, c'est celui que nous pouvons goûter toutes portes ouvertes, et qui sert à tous les hommes ${ }^{132}$.

57 En somme, il est clair qu'on ne peut reprocher à Emerson, malgré son admiration romantique pour les hommes supérieurs, de n'avoir pas mis en garde ses 
contemporains contre les duperies dont sauraient être capables certains Representative Men. Cet avertissement contre toute idolâtrie excessive de l'homme représentatif était déjà clairement proclamé dans l'introduction du même essai, lorsqu'il écrivait que: «La faiblesse des hommes invite toujours à l'impudence le pouvoir. Ce sont les délices d'un talent vulgaire d'éblouir et d'aveugler le spectateur. Mais le vrai génie cherche à nous défendre contre lui-même. Le vrai génie ne veut point appauvrir, mais délivrer, et ajouter de nouveaux sens ${ }^{133}$.» Toutefois, pas moins que Malaparte quelques décennies plus tard, le fait est qu'Emerson subit aussi la fascination de la force et du pouvoir viril de l'élite - celle des poètes ou de la politique -, seule capable de juguler la " populace » et de garantir l'intégrité et la puissance de la nation ; cela transparaît dans les pages d'essais comme Character ou Power ${ }^{134}$.

La pensée fluctuante, toujours mouvante, procédant par rotations (Circles), de Ralph Waldo Emerson a donc su séduire le jeune écrivain toscan en alimentant son désir absolu d'infini et de paix intérieure après la douloureuse épreuve de la guerre. Mais à mesure que Malaparte mûrit son engagement politique dans le fascisme, et accepte donc, du moins provisoirement, le recours à la violence politique, la figure du philosophe américain tend à pâlir. Moins de deux mois avant son adhésion au Fascio de Florence, dans les pages du journal La Rivoluzione liberale dirigé par Gobetti, Malaparte règle ses comptes avec le penseur américain, faisant de lui l'un des précurseurs de ce vague spiritualisme utilitariste qui serait propre aux États-Unis, reniant en quelque sorte ses propres élans panthéistes et universalistes, colonne vertébrale de l'expérience océanique. Aussi, imaginant une sorte d'idole géante américaine postée à cheval entre l'Asie et l'Europe, il écrit :

Soltanto questo mostruoso idolo di cemento armato potrebbe soddisfare il loro titanico e affannoso desiderio di divinità. Un dio imbevuto di teorie economiche e umanitarie, che avesse l'humour di Twain, l'ideologismo romantico di Emerson, l'asma panteistica di Whitman, le allucinazioni di Poe, la rudezza di Jack London e l'inventività di Edison [...]. Questa ricerca del divino è venuta prendendo fra gli americani, in questi ultimi anni, tutti i caratteri dell'ossessione. Al culto primitivo, importato dall'Inghilterra, degli eroi, dei «representative men» di Emerson, si era già sostituito da tempo il culto dell'uomo sociale, della volontà, dell'attività creatrice. Whitman era venuto ad annunziare $\mathrm{i}$ «re nuovi» $»^{135}$.

Cet antiaméricanisme que partageraient sans doute Gobetti et Gramsci aurait pu laisser la porte ouverte à une conversion libérale-révolutionnaire ou franchement communiste. Il n'en fut rien. Tournant le dos à Gobetti et à Emerson, sans pour autant partager les élans prométhéens des futuristes, Kurt Erich Suckert s'apprête à franchir le torrent impétueux qui le sépare encore des bataillons de chemises noires.

\section{NOTES}

1. C. E. Suckert, «A un fante», Oceanica, $n^{\circ} 3$, $1^{\text {er }}$ février 1921, p. 3. Suckert italianisait parfois l'orthographe de son prénom, «Kurt » devenant alors «Curt ». La poésie «A un fante » conjugue à la fois les "Correspondances" baudelairiennes et l'émanatisme de Swedenborg ou de son interprète américain, Emerson. 
2. Que le lecteur nous pardonne : bien que nous nous focalisions sur les années 1921-1923, soit un laps de temps relativement restreint, nous emploierons dans cette étude indistinctement Suckert et son pseudonyme littéraire, Malaparte, adopté en réalité fin 1924 seulement.

3. G. Pardini, Curzio Malaparte. Biografia politica, préface de F. Perfetti, Milan - Trente, Luni Editrice, 1998.

4. En effet, sous l'influence théorique de Georges Sorel, le syndicalisme révolutionnaire se développe en Italie dès le début $\mathrm{du} \mathrm{Xx}^{\mathrm{e}}$ siècle, autour des figures de proue que sont alors Arturo Labriola, les frères De Ambris ou Filippo Corridoni. En 1914, une partie du mouvement suivra Mussolini sur la voie de l'interventionnisme militaire, en rupture avec sa branche pacifiste, et, après la guerre, rejoindra les rangs fascistes. Voir W. Gianinazzi, «Le syndicalisme révolutionnaire en Italie (1904-1925) », Mil neuf cent, 2006/1, n² 24, p. 95-121.

5. F. Germinario, Sorel davanti al nazionalismo italiano, dans P. Pastori et G. Cavallari (éd.), Georges Sorel nella crisi del liberalismo europeo, Camerino, Università degli Studi di Camerino, 2001, p.319-332. Pour notre propos, il importe de préciser que les premières convergences significatives entre syndicalistes révolutionnaires et monarchistes nationalistes ont lieu dès 1911, à travers l'expérience du Cercle Proudhon, et n'excèdent pas, dans le cas de Sorel, 1914. Sur la genèse du Cercle, est toujours d'actualité l'étude de G. Navet, «Le Cercle Proudhon (1911-1914). Entre le syndicalisme révolutionnaire et l'Action française ", Mil neuf cent, 1992, vol. 10, nº 1, p. 46-63. Quant au Cercle entendu comme un "préfascisme ", cf. la réfutation convaincante de A. de Benoist, Préface à la réédition des Cahiers Proudhon, Nantes, Avatar Éditions, 2007, p. 81-86. Sur les convergences entre Sorel et les droites radicales, en dépit des vifs débats qu'ils ont provoqués, on ne peut que renvoyer aux travaux de Sternhell, notamment à Z. Sternhell, Georges Sorel, le syndicalisme révolutionnaire et la droite radicale au début du siècle, dans J. Julliard et $\mathrm{S}$. Sand (éd.), Georges Sorel en son temps, Paris, Seuil, 1985, p. 75-100.

6. Sur ce courant spiritualiste, voir P. Gura, American Transcendentalism. A History, New York, Hill \& Wang, 2007.

7. Ces deux auteurs ont pu marquer Malaparte en profondeur, en particulier quant à sa vision surréelle et transcendantale de la nature, voire dans la quête d'infini propre à l'océnanisme. Walden, le chef-d'œuvre de Thoreau, est traduit pour la première fois en italien la même année que Viva Caporetto!, à Florence, chez l'éditeur Battistelli; toutefois, il est peu probable que Malaparte l'ait lu dans cette édition puisque Viva Caporetto! est publié au tout début de l'année 1921. Peut-être l'a-t-il découvert plus tard, dans la continuité de son intérêt avéré pour Emerson. Il ne fait pas l'ombre d'un doute, en revanche, qu'il connaissait ce dernier, qu'il cite plusieurs fois, notamment le célèbre Representative Men, dont la version italienne, Uomini rappresentativi, est publiée en 1904 chez Fratelli Bocca, à Turin. Il est à remarquer qu'à partir de cette date, les traductions de textes d'Emerson - qui font leur apparition dans les bibliothèques du royaume dans les dernières décennies $d u \mathrm{XIX}^{\mathrm{e}}$ siècle - se multiplient en Italie. Sur les liens entre Emerson et Thoreau, voir J. T. Lysaker et W. Rossi, Emerson \& Thoreau. Figures of Friendship, Bloomington, Indiana University Press, 2010.

8. C. Malaparte, Viva caporetto! La rivolta dei santi maledetti, M. Biondi (éd.), Florence, Vallecchi, 1995 (1921), p. 49. Toutes les citations originales de l'ouvrage sont tirées de cette édition qui reproduit la princeps de 1921.

9. S. Laporte, Préface à C. Malaparte, Viva Caporetto!, Paris, Les Belles Lettres, 2012 (1921), p. 12.

10. C. Malaparte, Viva caporetto!, ouvr. cité, p. 49.

11. Ibid., p. 50.

12. Les quatre numéros d'Oceanica ont été publiés entre janvier et mars 1921. Voir la recherche de référence conduite par S. Laporte, L'Océanisme: une passion cosmopolite du jeune Malaparte à la veille de son adhésion au fascisme (1921-1922), dans U. Lemke, M. Lucarelli et E. Mattiato (éd.), Cosmopolitisme et réaction : le triangle France-Allemagne-Italie dans l'entre-deux-guerres, préface de G.- 
H. Soutou, Chambéry, Éditions de l'Université de Savoie Mont Blanc - LLSI, 2014, p. 229-256. Cf. aussi M. Serra, Malaparte. Vies et légende, Paris, Perrin, 2012 (2011), p. 93-104.

13. A. Colombo, Per una rilettura della Rivolta dei santi maledetti, dans G. Grana (éd.), Malaparte scrittore d'Europa. Atti del convegno (Prato 1987) e altri contributi, Milan, Marzorati, 1991, p. 180-181.

14. C. Malaparte, Viva Caporetto!, ouvr. cité, p. 49.

15. M. Lucarelli, «Introduzione » à M. Lucarelli et M. Mastroianni (éd.), Lo specchio, il doppio, la guerra: l'identità sdoppiata. Poetiche a confronto, "Quaderni di studi franco-italiani / Cahiers d'études franco-italiennes ", Alessandria, Edizioni dell'Orso, 2016, p. 131-136.

16. Qu'il me soit ici permis de renvoyer à E. Mattiato, « Messianismo politico e antimodernità nei primi miti letterari di Curzio Malaparte ", Revue des études italiennes, t. 55, janvier-juin 2009 (janvier 2012), no 1-2, Paris, L'Âge d'Homme / Université Paris-Sorbonne - Paris 4, p. 109-121. Notons au passage qu'il n'est point besoin d'être mazzinien et fasciste pour exalter le sacrifice politique : Piero Gobetti lui-même se réappropriera cette notion pour donner un sens libéral et marxien à sa lutte antifasciste, se percevant lui-même comme un prêtre se préparant au don de soi par la lutte politique. P. Gobetti, «Elogio della ghigliottina », La Rivoluzione Liberale, a. I, $\mathrm{n}^{\circ}$ 34, 23 novembre 1922, dans Opere complete di Piero Gobetti. Scritti politici, P. Spriano (éd.), vol. I, Turin, Einaudi, 1997 (1961), p. 433-434.

17. Malaparte reviendra à plusieurs reprises sur l'action bénéfique de Mussolini sur l'unité italienne, le fascisme apparaissant comme le parachèvement du processus unitaire, ne reniant ni Cavour, ni Mazzini, ni Garibaldi (contrairement, par exemple, à la désacralisation des deux derniers opérée par Piero Gobetti) : voir C. Malaparte, «Il rito dell'Italia fascista da Cavour a Mussolini », Il Corriere della Sera, 16 juin 1929; "Un'altra lezione», Il Corriere della Sera, 10 septembre 1929.

18. M. Gervasoni, L'intellettuale come eroe. Piero Gobetti e le culture del Novecento, Milan, La Nuova Italia, 2000, p. 216-217. Du même auteur, voir Gramsci e la Francia, Milan, Unicopli, 2000.

19. C. Malaparte, Viva Caporetto!, ouvr. cité, p. 49.

20. Ibid. C'est nous qui soulignons.

21. F. T. Marinetti, Fondazione e Manifesto del Futurismo (1909), dans Id., Teoria e invenzione futurista, L. De Maria (éd.), Milan, Mondadori, 1983 (1968), p. 8. C'est nous qui soulignons. S'il est entendu que ce texte fondateur connut une résonance mondiale grâce à sa publication en français en première page du Figaro le 20 février 1909, il n'en demeure pas moins qu'il a été publié en italien l'année précédente dans au moins deux gazettes provinciales (La Gazzetta di Mantova e La Gazzetta dell'Emilia), sans connaître, bien entendu, un quelconque retentissement.

22. «Bisogna » indique la nécessité, inhérente au programmatisme «astreignant » de quasiment tous les manifestes futuristes (il n'est pas surprenant que l'on retrouve souvent ce verbe dans les hendécalogues futuristes) ; «vita » est la sève vitaliste de l'élan futuriste ; enfin, la préposition «per» introduit une proposition finale implicite désignant l'objectif de la transhumanisation futuriste. Sans pouvoir analyser en détails la fonction des calques malapartiens, bornons-nous à constater qu'ils ont une signification fort différente de l'orientation marinettienne.

23. On nous rétorquera que Marinetti avait derrière lui une œuvre déjà riche de nombreux livres en français et de textes italiens. Soit, mais le Manifeste inaugure aussi une authentique seconde naissance littéraire.

24. «Bisogna » est un présent d'ordre général, idéal pour traduire l'horizontalité quotidienne de l'océanisme et s'oppose au futur programmatique de la citation marinettienne ; "aver disceso [...], sofferto, sperato, maledetto » est un renversement des élans ascensionnels et optimistes des futuristes ; les « uomini [...] semplicemente umani », soucieux de mordre aux racines mêmes de la vie, vont à l'encontre des pulsions prométhéennes du futurisme, etc.

25. Ibid., p. 8.

26. C. Malaparte, Viva Caporetto!, ouvr. cité, p. 147, 151.

27. F. T. Marinetti, Fondazione e Manifesto del Futurismo, ouvr. cité, p. 8. 
28. C. Malaparte, Viva Caporetto!, ouvr. cité, p. 79.

29. Il y a au moins quatre allusions au franciscanisme dans Viva Caporetto!, toutes reliées sémantiquement à l'abaissement, à la crasse des soldats, et à leur signification mystique. Dès l'incipit, il est fait mention de l'« uomo » (le narrateur, en réalité) sorti du cercle de la guerre «benedicendo Dio, a capo chino, come un francescano» (ibid., p. 49). À la fin du chapitre v, Suckert écrit au sujet de la puanteur de la soldatesque : «Quel tanfo eroico è quello dei santi, dei pellegrini, dei mistici, dei fratelli in Cristo ed in madonna Povertà: il poverello d'Assisi puzzava di lercio come un fante del Carso. » (Ibid., p. 91)

30. On comptabilise 25 occurrences de "Christ» et de ses dérivés dans Viva Caporetto!, exactement autant que "fango" et ses dérivés, deux lexèmes souvent associés et sémantiquement prééminents.

31. Paradoxalement, dans les dernières années de leur existence, on pourrait rapprocher Marinetti et Malaparte en prenant comme point de jonction leur commune fascination - particulièrement désacralisante dans le cas du premier - pour la figure du Christ : P. Valesio, Il dopo-Cristo fra Marinetti e Malaparte, dans R. Barilli et V. Baroncelli (éd.), Curzio Malaparte. Il narratore, il politologo, il cittadino di Prato e dell'Europa, Naples, CUEN, 2000, p. 93-106.

32. Ibid., p. 49.

33. Anonyme, "Nè passatismo nè futurismo: questa è la parola d'ordine degli 'Oceanici' ", Oceanica, $\mathrm{n}^{\circ}$ 2, 15 janvier 1921, p. 17.

34. F. T. Marinetti, « Manifesto tecnico della letteratura futurista. 11 maggio 1912 », in Id., Teoria e invenzione futurista, p. 54. Le projet philosophique d'Oceanica et de Viva Caporetto! se construit donc par contraste avec le futurisme, comme le remarque aussi, sans développer son intuition, Andrea Orsucci: A. Orsucci, Il «giocoliere d'idee». Malaparte e la filosofia, Pise, Edizioni della Normale, 2015, p. 52.

35. Cela a été justement signalé par Andrea Orsucci, lequel consacre aussi de belles pages à l'analyse du Christ-Homme malapartien, «Christ russe » dérivé tant des écrits de Berdiaev que de Soloviev. Cf. A. Orsucci, Il «giocoliere d'idee». Malaparte e la filosofia, ouvr. cité, p. 70. Il eût été fécond pour notre propos qu'Orsucci mît en relation concrètement Malaparte et Emerson, qui est cité à plusieurs reprises dans Viva Caporetto! Voici le texte original, nous reviendrons sur son interprétation : «It is natural to believe in great men. If the companions of our childhood should turn out to be heroes, and their condition regal it would not surprise us. All mythology opens with demigods, and the circumstance is high and poetic; that is, their genius is paramount. In the legends of the Gautama, the first men ate the earth and found it deliciously sweet. » (R. W. Emerson, Representative Men (1850), in Id., Complete Works 1904, Hollister, YOGeBooks, 2014, p. 723. C'est nous qui soulignons. Ce texte, consulté le 15 avril 2016, est en ligne sur: <www.yogebooks.com/ english/emerson/1904completeworks.pdf>.)

36. C. Malaparte, Viva Caporetto!, ouvr. cité, p. 49.

37. Le texte recense 18 occurrences de "umile» et de ses dérivés, toujours associés aux fantassins ou au narrateur en personne. Voir aussi les jeux de mots poétiques autour de l'humilité, de la terre et de la prière dans C. E. Suckert « A un fante », art. cité.

38. Ibid., p. 49.

39. Ibid., p. 85.

40. L. Jenny, Je suis la révolution. Histoire d'une métaphore (1830-1975), Paris, Belin, 2008, p. 43-48. Marco Gervasoni, à juste titre, voit dans la notion d'auto-engendrement l'un des éléments constitutifs de la modernité politique et du nouveau discours révolutionnaire au lendemain de la Grande Guerre : cf. M. Gervasoni, L'intellettuale come eroe, ouvr. cité, p. 213 et suiv.

41. C. Malaparte, Viva Caporetto!, ouvr. cité, p. 144.

42. Ibid., p. 147, 151.

43. Notons au passage que « uomo » et ses dérivés comptent 143 occurrences dans Viva Caporetto!, soit un peu moins que « guerra » et ses dérivés (141). 
44. F. T. Marinetti, Fondazione e Manifesto del Futurismo, ouvr. cité, p. 9.

45. Voir l'étude exhaustive de Serge Milan sur ce sujet: S. Milan, L'antiphilosophie du futurisme. Propagande, idéologie et concepts dans les manifestes de l'avant-garde italienne, Paris, L'Âge d'Homme, 2009.

46. Cette notion de tendance paraît essentielle car elle inaugure et conclut la «Lettera ». Si l'on considère que la quête de l'infini est le cœur de l'océanisme, on pense immédiatement à l'approche transcendantaliste de la «tendance naturelle » propre aux disciples de Ralph Waldo Emerson, dont le panthéisme s'avère très proche de celui de Malaparte. Chez Emerson, une tendance est toujours une orientation naturelle, biologique, vers une perfection qui à la fois englobe et dépasse l'homme : « Nous ne sommes que des tendances, ou plutôt des symptômes, et aucun de nous n'est complet. Nous ne faisons que toucher barre en passant, et humer la mousse de plusieurs existences. La rotation est la loi de la nature. » (R. W. Emerson, Hommes représentatifs, traduction de J. Izoulet et F. Roz, Paris, Bartillat, 2015, p. 27.) Sur la tendance comme destin, cf. R. Picon, Emerson. Le sublime ordinaire, Paris, CNRS Éditions, 2015, p. 265-266. Dans son manifeste de l'océanisme, Malaparte a une conception émersonienne des tendances ouvertes vers l'infini : «Le facoltà umane sono infinite nelle tendenze, limitate nella pratica. Lo spirito borghese [...] minaccia di amputare le possibilità umane di spazio e di illimite.» (C. E. Suckert, « Il manifesto dell'oceanismo », Oceanica, n 1 , $1^{\mathrm{er}}$ janvier 1921, dans Malaparte. Volume I. 1905-1926, E. Ronchi Suckert (éd.), Florence, Ponte alle Grazie, 1991, p. 189.) Tous ces concepts - facultés humaines infinies, tendances, espaces illimités - sont des notions fondamentales des thèses transcendantalistes défendues par Emerson.

47. C. E. Suckert, «Lettera ad un oceanico svizzero", Oceanica, $\mathrm{n}^{\circ} 3,1^{\mathrm{er}}$ février 1921 , dans Malaparte. Volume I. 1905-1926, ouvr. cité, p. 341. Je remercie Massimo Lucarelli de me l'avoir signalée ; et je suis infiniment reconnaissant à Stéphanie Laporte de m'avoir permis de consulter la revue dans sa version originale.

48. Ibid.

49. P.-A. Taguieff, Le nouveau national-populisme, Paris, CNRS Éditions, 2012, p. 47-49.

50. C. Malaparte, Viva Caporetto!, ouvr. cité, p. 49.

51. Sur les origines sans doute vociane de cette écologie essentialiste et sur ses linéaments, cf. I. Buttignon, Il verde e il nero. Maccari, Malaparte, Soffici: $i$ fascisti che anticiparono l'ambientalismo, Milan, Hobby \& Work, 2011, p. 87-108.

52. C. Malaparte, Viva Caporetto!, ouvr. cité, p. 76.

53. Le schème oppositif Peuple opprimé et bon versus élite oppressive et corrompue est opérant chez Malaparte dès son adhésion de jeunesse aux idéaux de Mazzini, modelés sur le Peuple italien souffrant et conformé au Christ ; c'est là sans doute, malgré ses mutations narratives, l'un des motifs les plus durables de la palette expressive malapartienne ; c'est aussi ce qui l'unit le plus, sans doute, à un autre intellectuel subversif, nostalgique d'une culture populaire préservée : Pier Paolo Pasolini. Sur cette scission manichéenne entre Peuple et élites propre au populisme, cf. P.A. Taguieff, L'illusion populiste. Essai sur les démagogies de l'âge démocratique, Paris, Flammarion, 2007, p. 19-22, 70-71.

54. Comme Malaparte après lui, Mussolini semble avoir basculé d'un populisme de gauche (sans équivoque possible, le "populisme » est, à sa naissance, de matrice socialiste) à un nationalpopulisme sous l'influence de Georges Sorel (du moins, d'un Sorel d'avant 1917, non encore tombé sous le charme de Lénine), dont nous avons déjà signalé l'importance dans l'essor de ce mouvement. Cf. P. Milza, "Mussolini entre fascisme et populisme», Vingtième Siècle, revue d'histoire, $\mathrm{n}^{\circ}$ 56, octobre-décembre 1997, p. 118.

55. Sur ce point, voir les trois volumes indépassables de F. Venturi, Il populismo russo, Turin, Einaudi, 1972 (1952). Sur la figure de Venturi, voir L. Casalino, Influire in un mondo ostile. Biografia politica di Franco Venturi (1931-1956), Aoste, Stylos, 2006. 
56. La question reste ouverte. Autant Marx fut attentif aux développements du populisme russe, autant Engels les considéra avec dédain après la mort de Marx. Si Malaparte a pu être indirectement influencé par les populistes russes, il ne faudrait pas négliger non plus le filon plus conservateur dont ce mouvement est issu, à savoir le mouvement romantique slavophile, dont l'influence se fait sentir jusqu'à la fin de l'Empire par le biais de Pobiedonostev. Cf. J. Detlev, Autocratie, orthodoxie et narodnost. Le conservatisme de Constantin Pobedonostsev au tournant des XIX et XX $x^{e}$ siècle, dans Marita Gilli (éd.), Les limites de siècles: champs de forces conservatrices et régressives depuis les temps modernes, vol. I, Besançon, Presses universitaires franc-comtoises, 2001, p. 186-187.

57. C. Malaparte, Viva Caporetto!, ouvr. cité, p. 51. C'est nous qui soulignons. Ce passage est en réalité la paraphrase d'un paragraphe tiré de la « Lettre à Daniel Halévy » de Georges Sorel, texte fondamental servant d'introduction à ses célèbres Réflexions sur la violence: il nous restera à démontrer - dans un article qui sera publié en 2017 dans les pages d'un numéro monographique de la revue Sinestesie consacré à Malaparte - que Réflexions sur la violence est la source matricielle de Viva Caporetto!, qui en constitue en quelque sorte une relecture autobiographique marquée par la guerre de 1914-1918. Cf. G. Sorel, Réflexions sur la violence, J. Julliard (éd.), Paris, Seuil, 1990, p. 21.

58. On pense immédiatement aux années de militance de Mussolini au Parti socialiste italien, à sa direction de l'Avantil, et, plus encore, au laboratoire politiques qu'a pu être l'éphémère revue (bien-nommée) Utopia, publiée entre novembre 1913 et décembre 1914.

59. E. Gentile, La Grande Italia. Il mito della nazione nel XX secolo, Rome-Bari, Laterza, 2011, p. 163.

60. La nation, pour Suckert, est moins un territoire ou une ethnie que le fruit du sacrifice des soldats ; on pourrait même aller jusqu'à dire que le vétéran, c'est l'Italie incarnée.

61. C. Malaparte, Memoriale (1946), cité dans M. Biondi, Malaparte e le guerre del Novecento, dans R. Barilli et V. Baroncelli (éd.), Curzio Malaparte, ouvr. cité, p. 226.

62. Voir par exemple R. W. Emerson, History, in Id., Essays - First Series (1841), in Id., Complete Works 1904, Hollister, YOGeBooks, 2014, p. 277-301. Ce texte est traduit en italien 70 ans plus tard: R. W. Emerson, L'anima, la natura e la saggezza. Saggi 1. e 2. serie, Bari, Gius. Laterza \& Figli, 1911.

63. C. Malaparte, Viva Caporetto!, ouvr. cité, p. 54.

64. Marx, au contraire, semble bien favorable à la disparition progressive de l'État une fois achevée la révolution: voir D. Collin, Le cauchemar de Marx. Le capitalisme est-il une histoire sans fin ?, Paris, Max Milo, 2009, p. 167-170, 266-267. S'il y a bien une critique du machinisme comme instrument d'aliénation de l'ouvrier, on chercherait en vain un Marx fourrier de la tradition et de l'État: non seulement parce qu'au nom de sa théorie abondanciste, la machine soumise au prolétariat doit, selon l'idéologie du "Progrès" continu propre au XIX ${ }^{\mathrm{e}}$ siècle, exploiter les ressources mondiales et assurer la justice sociale, mais aussi parce que l'État même n'a pas vocation à perdurer une fois dépassé le stade de la dictature du prolétariat; c'est la théorie engelsienne de l'« État dépérissant » qui fascinera tant Lénine, comme le rappelle Albert Camus dans L'homme révolté, Paris, Gallimard, 1951, p. 283. On trouve dans le Proudhon de De la justice, par exemple, des idées plus conformes à celles exprimées ici par Malaparte et attribuées à Marx : c'est ce Proudhon de l'ordre contre le chaos que tente de récupérer en 1912 le Cercle Proudhon, animé entre autres par Georges Valois: aventure sans issue politique, comme le sera le syndicalisme révolutionnaire malapartien après 1922 ; mais qui marquera profondément l'aile révolutionnaire du fascisme. Cf. par exemple P. Galland, « Proudhon et l'ordre ", Premier Cahier, janvier-février 1912, dans Cahiers du Cercle Proudhon, ouvr. cité, p. 133-136, ou encore J. Lagrange, « Proudhon et l'ordre européen », ibid., p. 175-185.

65. On a souvent taxé les syndical-révolutionnaires italiens de «libéristes de classe » dans la mesure où ils opposaient à l'État bourgeois et aux partis politiques les syndicats ouvriers. Certes, mais cela ne signifiait pas forcément la disparition de l'État; au contraire, l'État passé par l'épreuve de la révolution syndicale ne formerait plus qu'un avec les producteurs réunis. C'est 
l'embryon du corporatisme fasciste. Notons, pour actualiser notre propos, que ces dernières années, il est possible, en Italie, de se confronter à une relecture de Marx suivant une optique syndicale-révolutionnaire, nationale et anti-sociétaliste à partir des œuvres de Costanzo Preve ou de Diego Fusaro, son jeune et médiatique disciple. Georges Sorel, en revanche, était persuadé que Marx souhaitait la disparition de l'État et lui-même (du moins en 1908) semblait incliner vers le "dépérissement de l'État». Cf. G. Sorel, La décomposition du marxisme (1908), dans Id., La décomposition du marxisme et autres essais, T. Paquot (éd.), Paris, PUF, 1982, p. 244-249 ; G. Sorel, Réflexions sur la violence, Paris, Seuil, 1990, p. 108-113. Sur cette question, voir aussi P. King, Fear of Power. An Analysis of Anti-Statism in Three French Writers: Alexis de Tocqueville, Pierre-Joseph Proudhon, Georges Sorel, Londres, Frank Cass, 1967.

66. C. Malaparte, Viva Caporetto!, ouvr. cité, p. 57.

67. Sur l'essentialisation de Mussolini, très tôt (auto-)perçu comme homme de la Providence au destin politique hors du commun, cf. A. Tarquini, Storia della cultura fascista, Bologne, Il Mulino, 2011, p. 109-114 ; voir aussi D. Musiedlak, « Mussolini : le grand dessein à l'épreuve de la réalité », Parlement(s). Revue d'histoire politique, $\mathrm{n}^{\circ}$ 13, 2010/I, p. 51-62.

68. M. Onfray, La construction du Surhomme. D'Emerson et Carlyle à Burckhardt et Guyau, Vincennes, Frémeaux \& associés, 2010. Il s'agit de leçons enregistrées sur 12 disques compacts.

69. On pourrait remonter à la naissance de l'absolutisme royal, mais il ne fait pas de doute que la décapitation à la fois réelle et symbolique du dernier roi de France a ouvert le champ à l'émergence de figures prométhéennes dans la vie politique française. Les tentations autoritaires qui perturbent son histoire récente sont peut-être en soi moins une conséquence qu'une cause de la fin du royaume capétien. Voir J. Garrigues, Les hommes providentiels. Histoire d'une fascination française, Paris, Seuil, 2012.

70. Ibid., p. 121-125.

71. N. Dolan, Emerson's Liberalism, Madison, The University of Wisconsin Press, 2009, p. 236-237.

72. Le fait que ce texte serve de préambule à La rivolta dei santi maledetti est rarement signalé (hormis par Martellini); or, ce point est essentiel car, par sa position même, il impose une relecture orientée du texte original de 1921, lui-même partiellement modifié.

73. Soit l'une des trois catégories des droites françaises rendues célèbres par René Rémond et qui rejoint, certes partiellement, les questionnements de Suckert entre 1921 et 1924.

74. C. Malaparte, Ritratto delle cose d'Italia, degli eroi, del popolo, degli avvenimenti, delle esperienze e inquietudini della nostra generazione, dans Id., Viva Caporetto!, ouvr. cité, p. 157. Plus loin, les Representative Men en sont réduits à des « nani vociatori » (ibid., p. 165). Le nain est sans doute une image tirée d'Ainsi parlait Zarathoustra de Nietzsche.

75. Ibid., p. 155. Cette invocation à la tyrannie du héros est déjà au cœur de C. Suckert, L'Europa vivente. Teoria storica del sindacalismo nazionale, Florence, La Voce, 1923. Sur Mussolini comme héros "fisicamente e storicamente avverso agli italiani d'oggi», cf. C. E. Suckert, "Ritratto storico di Mussolini », La Nazione, 19 juin 1923, dans Malaparte. Volume I. 1905-1926, ouvr. cité, p. 341.

76. C. Malaparte, Viva Caporetto!, ouvr. cité, p. 59.

77. Ibid., p. 60.

78. C. Burrafato, L'Italia e la Francia di Antonio Aniante: la pubblicazione del «Mussolini» e il tentativo di riconversione di un originale perduto, mémoire de Master sous la direction de E. Mattiato, Université Savoie Mont Blanc - Università degli Studi di Torino, 2014-2015.

79. C. Malaparte, Viva Caporetto!, ouvr. cité, p. 60.

80. Sur cette épineuse question, voir l'analyse toute en nuances de W. Gianinazzi, « Georges Sorel et "le mystère" de la sélection morale des "autorités sociales" aux minorités agissantes ", Revue française d'histoire des idées politiques, 2005/2, n 22, p. 77-93.

81. Même après son adhésion officielle au fascisme, Malaparte reste fidèle aux thèses de Sorel sur la violence syndicale et, paraphrasant Filippo Corridoni, il défend un syndicalisme national qui 
conserve l'esprit de la bourgeoisie patriote du Risorgimento, dont l'héritage révolutionnaire serait passé au prolétariat. Cf. C. E. Suckert, « Il mito della violenza sindacalista (prima parte)», La Nazione, 7 février 1923, dans Malaparte. Volume I. 1905-1926, ouvr. cité, p. 330-331. Cette série d'articles sera ensuite intégrée à L'Europa vivente, essai publié la même année. Pour Malaparte, en 1924, Corridoni apparaît (presque neuf ans après sa mort sur le front) comme la figure napoléonienne et mythique prophétisée par Sorel, celle qui aurait pu sauver l'Italie. Voir C. Suckert, "Filippo Corridoni, martire operaio ", La Conquista dello Stato, $1^{\mathrm{er}}$ octobre 1924, dans Malaparte. Volume I. 1905-1926, ouvr. cité, p. 433-435.

82. Gobetti ironisera sur cette attente fasciste et moderne d'un héros providentiel. Il le fera en décochant, à deux reprises, ses flèches contre Marinetti et Mussolini. Dans un portrait au vitriol, le fondateur du futurisme est vu comme «l'uomo rappresentativo dell'epoca » (P. Gobetti, «Marinetti, il precursore », Il Lavoro, 31 janvier 1924, dans Opere complete di Piero Gobetti. Scritti politici, vol. I, ouvr. cité, p.580). Similairement, Mussolini est accusé d'avoir été «l'uomo rappresentativo di questa stanchezza e di questa aspirazione al riposo " qui permirent l'avènement du fascisme (P. Gobetti, La rivoluzione liberale. Saggio sulla lotta politica in Italia (1924), dans Scritti politici, ouvr. cité, p. 1074).

83. C. Suckert, "Il paradosso della lotta di classe», Camicia Nera, 30 septembre 1922, dans Malaparte. Volume I. 1905-1926, ouvr. cité, p. 287-288.

84. Ibid., p. 288. Ces mots ont un sens particulier si l'on se souvient que Malaparte adhère à cette époque au PNF précisément pour poursuivre une action syndicale-révolutionnaire, en prenant la direction de la Camera Italiana del Lavoro de Florence. Voir la série d'articles sur ce sujet dans ibid., p. 290 et suiv.

85. C. Malaparte, Viva Caporetto!, ouvr. cité, p.59. Sur la notion de latinité et son bassin sémantique, récupérés plus tard par le fascisme, voir C. Fraixe, L. Piccioni et C. Poupault (dir.), Vers une Europe latine. Acteurs et enjeux des échanges culturels entre la France et l'Italie fasciste, ParisBruxelles, INHA-Peter Lang, 2014.

86. P. Gobetti, La rivoluzione liberale, ouvr. cité, p. 933-937. Voir aussi le Risorgimento laborieux et silencieux, proprement antihéroïque (et antirhétorique), présenté dans P. Gobetti, Risorgimento senza eroi. Studi sul pensiero piemontese nel Risorgimento(1926), Rome, Edizioni di storia e letteratura, 20112. Sur l'héroïsme gobettien (proche de celui de Malaparte en vertu de la médiation sorélienne) et sur les intellectuels qui ont pu l'influencer, cf. l'excellente synthèse de M. Gervasoni, L'intellettuale come eroe, ouvr. cité, p. 213-252.

87. Cet ouvrage inachevé fut suffisamment important pour alimenter plusieurs nouvelles de Malaparte dans les années 1930. Cf. B. Baglivo, I racconti di Curzio Malaparte, mémoire de Master en littérature comparée, sous la direction de B. Manetti, Università degli Studi di Torino - Université Savoie Mont Blanc, 2014-2015, p. 17-18. Ce mémoire de Master est une contribution considérable pour comprendre la genèse des nouvelles de fiction publiées par l'auteur de la fin des années 1920 aux années 1940, phase de transition entre les écrits politiques de jeunesse et les grands romans de la maturité.

88. Il n'en reste pas moins que Sorel est l'une des références largement partagées tant par Malaparte que Gobetti ; c'est même le véritable trait d'union entre les deux hommes. Voir les pertinentes observations d'Arianna Camerano sur le sujet dans le présent recueil.

89. C. Malaparte, Viva Caporetto!, ouvr. cité, p. 75.

90. C'est cette palingénésie à laquelle aspirait Malaparte lui-même lorsqu'il pensait que le fascisme abolirait toutes les classes en donnant naissance à « una nuova civiltà: e siamo certi ch'essa non sarà né borghese né proletaria. Abbiamo l'una e l'altra ugualmente in odio » (c. Suckert, L'Europa vivente, ouvr. cité, p. 116).

91. C. Malaparte, Viva Caporetto!, ouvr. cité, p. 59-60. Mussolini comme Suckert pouvait trouver dans les écrits de Georges Sorel une critique acharnée des partis politiques et de la vie parlementaire. 
92. Ibid., p. 61. Ces mêmes masses sont immédiatement réhabilitées lorsqu'est évoquée la guerre italo-turque et qu'elles réagissent pour défendre leur « razza » et leur « sangue » (ibid., p. 62).

93. Ibid., p. 61.

94. Ibid., p. 67. Tout en chantant la révolte de Caporetto, la rébellion du soldat avide de paix, le narrateur ne cesse de revendiquer la gloire des batailles passées. C'est sans doute le paradoxe le plus fort du texte. Cf. ibid., p. 135.

95. Le peuple italien, « a parte certi difetti fondamentali di razza e di educazione, aveva saputo custodire intatti alcuni fra i principi di morale su cui poggiano la famiglia e lo stato » (ibid., p. 61). 96. « Nous disons aux étrangers [...] que la magnifique originalité de notre race vient d'éclater dans l'oCÉANISME, le plus moderne mouvement artistique européen destiné à grouper, en son nom, les hommes libres de tous les pays pour la renaissance de l'art et du sens universel de la vie. QUE LES ÉTRANGERS N'OUBLIENT JAMAIS, même si l'océanisme allait être de suite imité à Paris ou bien à Tipparery, que la première parole en est partie d'Italie et que l'océanisme reste et restera un mouvement international d'origine italienne." (Anonyme, "Nous disons aux étrangers ", Oceanica, $\mathrm{n}^{\mathrm{o}} 1,1^{\mathrm{er}}$ janvier 1921, p. 28, en annexe de S. Laporte, «L'Océanisme », art. cité, p. 255.)

97. Tentant un rapprochement progressif entre la condition des soldats autrichiens et celle des Italiens, Suckert conclut : "Noi siamo un popolo di proletarî che sanno capire anche i proletarî degli altri paesi: per noi la patria non è una questione di razze e di civiltà contrarie.» (C. Malaparte, Viva Caporetto!, ouvr. cité, p. 81.)

98. Sur ce point délicat, Maurizio Serra a fait une synthèse très précise et, à juste titre, interprète la conversion fasciste de Malaparte comme un pari politique pour la conquête du pouvoir (M. Serra, Malaparte, ouvr. cité, p. 105-116).

99. C. Malaparte, Viva Caporetto!, ouvr. cité, p.57. Ce héros était explicitement assimilé à l'« homme représentatif » d'Emerson. C'est là une interprétation abusive ou, pour le moins, très partielle du Representative Man émersonien. Nous y reviendrons.

100. Ibid., p. 61.

101. Ibid., p. 139-140.

102. M. Serra, Malaparte, ouvr. cité, p. 97.

103. C'est la question du «nazionale-popolare », au sens gramscien, dont la finalité est, in fine, toujours l'internationalisme. Cf. A. Gramsci, Quaderni del carcere, Turin, Einaudi, 1975, Q.14, p. 1728-1730. Voir aussi D. Fusaro, Gramsci. La passione di essere nel mondo, Turin, Feltrinelli, 2015.

104. C. E. Suckert, « Lettera ad un oceanico svizzero », art. cité.

105. Dans ce même ordre d'idées, on trouve maintes affinités entre la notion émersonienne d'«ordinaire» et le goût proclamé par le Malaparte de 1921 pour les bonheurs les plus rudimentaires de la vie, à commencer par les miracles de la nature et la simplicité du bon peuple. Sur le concept éminemment politique de l'ordinaire chez Emerson, cf. S. Laugier, Une autre pensée politique américaine. La démocratie radicale d'Emerson à Stanley Cavell, Paris, M. Houdiard, 2004, p. 20-24.

106. R. W. Emerson, Hommes représentatifs, ouvr. cité, p. 17.

107. Ibid., p. 21-22.

108. D. O. Dowling, Emerson's Protégés. Mentoring and Marketing Transcendentalism's Futur, New Haven, Yale University Press, 2014.

109. A. Versluis, American Gurus. From American Transcendentalism to New Age Religion, New YorkOxford, Oxford University Press, 2014.

110. Le Manifeste débute ainsi : "L'essenza delle concezioni umane è in pericolo. Il senso del frammentario, del particolare, del limitato, tende a sminuzzare l'antico senso oceanico della vita. » (C. E. Suckert, «Il manifesto dell'oceanismo », art. cité, p. 189.) On remarquera que la seconde partie du $1^{\mathrm{er}}$ chapitre de Viva Caporetto! fait des emprunts textuels à ce manifeste et à d'autres articles d'Oceanica. L'on songe notamment à l'idée d'encerclement létal (transposé du champ de bataille à l'humaine condition), ou encore à l'aveuglement des hommes face à l'infini, 
comparé à celui de Tobit, de biblique mémoire, que l'on retrouve dans Gli oceanici [K. E. Suckert], «Appello a tutti i ricercatori di Dio », Oceanica, n 4, mars 1921, dans Malaparte. Volume I. 1905-1926, ouvr. cité, p. 198. La critique de la « fragmentarité » était commune dans les pages de La Voce, la revue fondée en 1908 par Giuseppe Prezzolini. Cf. S. Laporte, « L’Océanisme », art. cité, p. 239.

111. C. Malaparte, Viva Caporetto!, ouvr. cité, p. 52.

112. Ibid., p. 55.

113. R. W. Emerson, Nature, Addresses and Lectures (1849), in Id., Complete Works 1904, ouvr. cité, p. 107, 128.

114. R. W. Emerson, Hommes représentatifs, ouvr. cité, p. 54. Malaparte mentionne aussi la « divina essenza » dans sa poésie très émersonienne «Ad un fante » (publiée début 1921 dans le $3^{\mathrm{e}}$ numéro d'Oceanica), dont nous avons cité un extrait en épigraphe de cette étude.

115. R. W. Emerson, Over-Soul (1841), in Id., Complete Works 1904, ouvr. cité, p. 456. Ce terme est généralement traduit en français par «âme supérieure » ou "âme suprême ». L'ouvrage est traduit en italien la même année que Reprensentative Men : R. W. Emerson, Saggi. L'anima suprema, l'amore, l'amicizia, la politica, Milan, L. F. Pallestrini \& C., 1904.

116. A. Versluis, American Transcendentalism and Asian Religions, New York-Oxford, Oxford University Press, 1993.

117. R. W. Emerson, Nature (1836), in Id., Complete Works 1904, ouvr. cité, p. 9.

118. Gli oceanici [K. E. Suckert], « Appello a tutti i ricercatori di Dio », art. cité, p. 198-199.

119. L'intuition est la faculté centrale accompagnant la quête philosophique et spirituelle des transcendantalistes. Malaparte a pu emprunter ces concepts non seulement à Emerson mais aussi à Bergson, plus proche de lui dans le temps et alors très à la mode. Cf. entre autres R. W. Emerson, Nature, Addresses and Lectures, ouvr. cité, p. 199, 236 ; Id., Self-Reliance, in Id., Essays - First Series, in Id., Complete Works 1904, ouvr. cité, p. 317 et surtout p. 496. Cf. aussi P. J. Keane, Emerson, Romanticism, and Intuitive Reason. The Transatlantic "Light of all our Day", Columbia-Londres, University of Missouri Press, 2005, p. 46-79.

120. Emerson prêche l'émancipation du moi et le non-conformisme absolu, l'histoire elle-même ne pouvant se comprendre que par la médiation du Self. Nous avions souligné que cette doctrine avait tout pour séduire le jeune Suckert, avide de faire coïncider dans Viva Caporetto! à la fois la grande Histoire, celle du peuple et la sienne propre. Cela dit, Thomas Constantinesco démontre avec brio que les théories du moi développées par Emerson pouvaient être mouvantes, voire contradictoires, son discours le plus mature n'exaltant en rien l'égotisme en dépit du recours au Je, et tendant de fait à l'impersonnalité (cf. T. Constantinesco, Ralph Waldo Emerson. L'Amérique à l'essai, Paris, Éditions Rue d'Ulm, 2012, p. 125-152).

121. W. T. Mott, Ralph Waldo Emerson in Context, New York, Cambridge University Press, 2014, p. 131-132. Pour plus de détails, cf. A.-M. Tuchinsky, "More Anon": American Socialism and Margaret Fuller's 1848, dans B. Bailey, K. P. Viens et C. E. Wright (éd.), Margareth Fuller and Her Circles, Durham, University of New Hampshire Press, 2013, p. 100-127.

122. R. W. Emerson, Nature, Addresses and Lectures, ouvr. cité, p. 103.

123. J'ai rappelé ailleurs que ce souffle océanique n'est pas une invention proprement malapartienne mais qu'on peut en relever la trace dans les écrits du pacifiste Romain Rolland (le «souffle océanique » comme nouvelle religion laïque) et, dans une moindre mesure, dans le cérébrisme de Ricciotto Canudo. Tous sont héritiers d'Emerson, de Baudelaire et des symbolistes. Cf. E. Mattiato, Paul Morand e Malaparte. Le tentazioni del cosmopolitismo, dans M. Grassi (éd.), La «Bourse des idées du monde». Malaparte e la Francia, Florence, Leo Olschki Editore, 2008, p. 128-129.

124. R. W. Emerson, Hommes représentatifs, ouvr. cité, p. 29.

125. Ibid., p. 155.

126. Ibid., p. 152.

127. C. Malaparte, Viva Caporetto!, ouvr. cité, p. 62.

128. Ibid., p. 139. 
129. R. W. Emerson, Hommes représentatifs, ouvr. cité, p. 161.

130. C. Malaparte, Viva Caporetto!, ouvr. cité, p.57. Nous avons largement commenté cette citation.

131. R.W. Emerson, Hommes représentatifs, ouvr. cité, p. 156. La métaphore du fer est déjà employée dans le premier chapitre des Hommes représentatifs, lorsqu'il exalte « un maitre qui se tient ferme sur des jambes de fer, bien né, riche, harmonieux de corps, éloquent, comblé d'avantages, entraînant tous les hommes par la fascination, pour en faire des tributaires et des soutiens de sa puissance. Épée et bâton, ou talents de même nature que l'épée ou le bâton, font l'œuvre du monde » (ibid., p. 29-30).

132. Ibid., p. 172.

133. Ibid., p. 27.

134. C. Constantinesco, Ralph Waldo Emerson. L'Amérique à l'essai, ouvr. cité, p. 227-233. Cf. aussi R. Picon, Emerson. Le sublime ordinaire, ouvr. cité, p. 263-266.

135. C. E. Suckert, «Le ultime eresie d'Occidente. Il desiderio anglo-sassone del divino", La Rivoluzione Liberale, 13 agosto 1922, dans Malaparte. Volume I. 1905-1926, ouvr. cité, p. 262.

\section{RÉSUMÉS}

Cette recherche explore les multiples indices intertextuels disséminés dans les pages de Viva Caporetto! - le premier ouvrage de Malaparte, qui contient en outre tous les mythes littéraires propres à sa poétique - et se propose de dépasser le simple discours sur la guerre et le pacifisme en faisant ressortir à la fois les éléments préfascistes de ce texte et, plus profondément, l'influence spirituelle du philosophe américain Ralph Waldo Emerson, la tentation de l'avantgarde futuriste (en particulier lorsque le jeune auteur renverse quelques stéréotypes marinettiens) et le dialogue contrastif avec son ami et adversaire politique Piero Gobetti.

La presente ricerca esplora le varie spie intertestuali sparse nelle pagine di Viva Caporetto! - la prima opera di Malaparte, contenente inoltre tutti i miti letterari caratteristici della sua poetica - e intende superare il mero discorso su guerra e pacifismo mettendo in risalto sia gli elementi prefascisti di tale testo che, più profondamente, l'influenza spirituale del filosofo americano Ralph Waldo Emerson, la tentazione dell'avanguardia futurista (in particolare quando il giovane autore ribalta alcuni stereotipi marinettiani) e il dialogo contrastivo con il suo amico e avversario politico Piero Gobetti.

Viva Caporetto! is Malaparte's first book but one finds already in it the main literary myths of his poetics. This essay explores the numerous inter-textual traces to be found in the book and deals more specifically with its pre-fascist elements, with Emerson's influence on Malaparte, with his reversal of some of Marinetti's stereotypes and with his agonistic dialogue with Piero Gobetti, who was both his friend and his political opponent. 


\section{INDEX}

Keywords : Curzio Malaparte, Filippo Tommaso Marinetti, Piero Gobetti, Ralph Waldo Emerson, Georges Sorel, fascism, pre-fascism, Risorgimento, Avant-gardes, manifestos, First World War, Viva Caporetto!, Caporetto, worship of the leader, transcendentalism, Cesarism, myth, political myth, literature and philosophy

Parole chiave : Curzio Malaparte, Filippo Tommaso Marinetti, Piero Gobetti, Ralph Waldo Emerson, Georges Sorel, fascismo, prefascismo, Risorgimento, avanguardie, manifesti, Prima guerra mondiale, Viva Caporetto!, Caporetto, culto del capo, trascendentalismo, cesarismo, mito, mito politico, letteratura e filosofia

Mots-clés : Curzio Malaparte, Filippo Tommaso Marinetti, Piero Gobetti, Ralph Waldo Emerson, Georges Sorel, fascisme, préfascisme, Risorgimento, avant-gardes, manifestes, Première Guerre mondiale, Viva Caporetto!, Caporetto, culte du chef, transcendantalisme, césarisme, mythe, mythe politique, littérature et philosophie

\section{AUTEUR}

\section{EMMANUEL MATTIATO}

Université Savoie Mont Blanc 